\title{
Approximations of Stochastic Navier-Stokes Equations
}

\author{
Shijie Shang $^{1} \quad$ Tusheng Zhang ${ }^{2}$
}

September 28, 2017

\begin{abstract}
In this paper we show that solutions of two-dimensional stochastic Navier-Stokes equations driven by Brownian motion can be approximated by stochastic Navier-Stokes equations forced by pure jump noise/random kicks.
\end{abstract}

Key words: Stochastic Navier-Stokes equations; Stochastic partial differential equations; Approximations; Weak convergence; Jump noise

AMS Subject Classification: Primary 60H15 Secondary 93E20, 35R60.

\section{Introduction}

Stochastic Navier-Stokes equations (SNSEs) are now a widely accepted model for fluid motion with random perturbations. In this paper, we consider the two-dimensional stochastic Navier-Stokes equations with Dirichlet boundary conditions on a bounded domain, which is given as follows:

$$
\begin{cases}d u-\kappa \Delta u d t+(u \cdot \nabla) u d t+\nabla \mathfrak{P} d t=F(u) d t+\sum_{i=1}^{m} \sigma^{i}(u) d W^{i}, & \text { in } \mathcal{O} \times(0, \mathrm{~T}], \\ \operatorname{div} u=0 & \text { in } \mathcal{O} \times(0, \mathrm{~T}], \\ u=0 & \text { in } \partial \mathcal{O} \times(0, \mathrm{~T}], \\ u(0)=h & \text { in } \mathcal{O},\end{cases}
$$

${ }^{1}$ School of Mathematical Sciences, University of Science and Technology of China, 230026 Hefei, China. Email: ssjln@mail.ustc.edu.cn

${ }^{2}$ School of Mathematics, University of Manchester, Oxford Road, Manchester M13 9PL, England, U.K. Email: tusheng.zhang@manchester.ac.uk 
where $\mathcal{O}$ is a bounded domain of $\mathbb{R}^{2}$ with boundary $\partial \mathcal{O}$ of class $\mathcal{C}^{3}$. $u=$ $\left(u_{1}, u_{2}\right)$ and $\mathfrak{P}$ represent the random velocity and modified pressure, respectively. $\kappa$ is the kinematic viscosity, for simplicity, we let $\kappa=1$ in this paper. $W=\left(W^{1}(t), \cdots, W^{m}(t)\right)$ is a $m$-dimensional standard Brownian motion. The fluid is driven by external force $F(u) d t$ and the random noise $\sum_{i=1}^{m} \sigma^{i}(u) d W^{i}$.

Stochastic Navier-Stokes equations have been studied by many people. There is a great amount of literature. Let us mention a few. SNSEs driven by white noise in time were first studied by Bensoussan and Temam in [2]. The existence and uniqueness of solutions of 2-D SNSEs driven by Lévy noise were obtained in [4], large derivation and moderate deviation principles were established in $[14,6]$. The ergodic properties and invariant measures of the 2-D SNSEs were studied in [9] and [8].

The aim of this paper is to study the approximations of SNSEs in (1.1) by SNSEs forced by Poisson random measures. One of the motivations is to shine some light on numerical simulations of SNSEs driven by pure jump noise. Recently, Nunno and Zhang in [5] obtained such an approximation for a general class of SPDEs. However, the results in [5] couldn't cover the stochastic Navier-Stokes equations, an important model in fluid dynamics. The difficulty lies in establishing the tightness of the approximating equations in the space of Hilbert space-valued right continuous paths with left limits. To overcome this difficulty, we first assume that the initial value has higher regularity, the external force and the coefficients of the jump noise take values in a more regular space, so that we can derive an uniform estimate of the stronger norm of the approximating solutions. With these estimates, we are able to prove the tightness of the approximating equations by Aldou's criterion, then through martingale characterization we show that the limit of the solutions of approximating equations is the solution of the SNSE driven by Brownian motion. We emphasize that the method of establishing the tightness here is different and simpler than that used in [5]. In the second step, we are able to remove the regularity restrictions on the coefficients and the initial condition by using finite dimensional approximations and establishing some uniform convergence in probability of the approximating solutions. In the final part of the paper, we provide several illustrating examples.

The rest of the paper is organized as follows. In Section 2 we lay down the precise framework. The main part is Section 3, where the approximations are established. In Section 4 some examples are provided. 


\section{Framework}

Let $(\Omega, \mathcal{F}, P)$ be a probability space equipped with a filtration $\left\{\mathcal{F}_{t}\right\}_{t \geq 0}$ satisfying the usual conditions. $\nu^{i}(d x), i=1, \cdots, m$ denote $\sigma$-finite measures on the measurable space $\left(\mathbb{R}_{0}, \mathcal{B}\left(\mathbb{R}_{0}\right)\right)$, where $\mathbb{R}_{0}:=\mathbb{R} \backslash\{0\}$. Let $N^{i}, i=1, \cdots, m$ be mutually independent $\mathcal{F}_{t}$-Poisson random measures on $[0, T] \times \mathbb{R}_{0}$ with intensity measure $d t \times \nu^{i}(d z)$ respectively. For $U \in \mathcal{B}\left(\mathbb{R}_{0}\right)$ with $\nu^{i}(U)<\infty$, we write

$$
\tilde{N}^{i}((0, t] \times U):=N^{i}((0, t] \times U)-t \nu^{i}(U), \quad t \geq 0,
$$

for the corresponding compensated Poisson random measures on $[0, T] \times \Omega \times$ $\mathbb{R}_{0}$. See [10] for the details on Poisson random measures.

We introduce the following standard space

$$
V=\left\{u \in H^{1}(\mathcal{O})^{2}: \nabla \cdot u=0,\left.u\right|_{\partial \mathcal{O}}=0\right\},
$$

with the norm $\|u\|_{V}:=\left(\int_{\mathcal{O}}|\nabla u|^{2} d x\right)^{1 / 2}$ and the inner product $((\cdot, \cdot))$. Denote by $H$ the closure of $V$ in the $L^{2}$-norm $\|u\|_{H}:=\left(\int_{\mathcal{O}}|u|^{2} d x\right)^{1 / 2}$. The inner product on $H$ will be denoted by $(\cdot, \cdot)$.

Identifying the Hilbert space $H$ with its dual space $H^{*}$, via the Riesz representation, we consider the system (1.1) in the framework of Gelfand triple:

$$
V \subset H \cong H^{*} \subset V^{*}
$$

We also denote by $\langle\cdot, \cdot\rangle$ the dual pair between $V^{*}$ and $V$ from now on.

Define the Stokes operator by

$$
A u:=-P_{H} \Delta u, \quad u \in D(A):=H^{2}(\mathcal{O})^{2} \cap V,
$$

where $P_{H}: L^{2}(\mathcal{O})^{2} \longrightarrow H$ is the usual Helmholtz-Leray projection. Actually, the map $A$ is an isomorphism between $V$ and $V^{*}$, and

$$
\langle A u, v\rangle=\langle u, A v\rangle=((u, v)), \quad \forall u, v \in V .
$$

Note that $\|A u\|_{H}$ is a norm on $V \cap H^{2}(\mathcal{O})^{2}$ which is equivalent to the Sobolev norm in $H^{2}(\mathcal{O})^{2}$ (for simplicity denoted by $H^{2}$ from now on), see Lemma III.3.7 in [13]. It is known that there exist an orthonormal basis $\left\{e_{i}, i \in \mathbb{N}\right\}$ in $H$ and corresponding eigenvalues $0<\lambda_{i} \uparrow<\infty$, that is

$$
A e_{i}=\lambda_{i} e_{i}, \quad i \in \mathbb{N} .
$$


Since the boundary $\partial \mathcal{O}$ is of class $\mathcal{C}^{3}$, it follows from Chapter I.2.6 in [13] that

$$
e_{i} \in H^{3}(\mathcal{O})
$$

Set

$$
b(u, v, w):=\sum_{i, j}^{2} \int_{\mathcal{O}} u_{i} \partial_{i} v_{j} w_{j} d x, \quad u, v, w \in V .
$$

Using integration by parts, it is easy to see that

$$
b(u, v, w)=-b(u, w, v), \quad b(u, v, v)=0, \quad u, v, w \in V .
$$

Throughout the paper, we will denote various generic positive constants by the same letter $C$, although the constants may differ from line to line. We now list some well-known estimates for $b$ which will be used in the sequel(see [13] for example):

$$
\begin{aligned}
& |b(u, v, w)| \leq 2\|u\|_{H}^{\frac{1}{2}}\|u\|_{V}^{\frac{1}{2}}\|w\|_{H}^{\frac{1}{2}}\|w\|_{V}^{\frac{1}{2}}\|v\|_{V}, \quad u, v, w \in V, \\
& |b(u, u, v)| \leq C\|u\|_{H^{2}}^{\frac{1}{2}}\|u\|_{V}\|u\|_{H}^{\frac{1}{2}}\|v\|_{H}, \quad u \in V \cap H^{2}, \quad v \in H .
\end{aligned}
$$

For $u, v \in V$, we denote by $B(u, v)$ the element of $V^{*}$ defined by

$$
\langle B(u, v), w\rangle=b(u, v, w), \forall w \in V .
$$

Therefore,

$$
\|B(u, v)\|_{V^{*}}=\sup _{\|w\|_{V} \leq 1}|b(u, v, w)| \leq 2\|u\|_{H}^{\frac{1}{2}}\|u\|_{V}^{\frac{1}{2}}\|v\|_{H}^{\frac{1}{2}}\|v\|_{V}^{\frac{1}{2}}
$$

hence

$$
\|B(u, u)\|_{V^{*}} \leq 2\|u\|_{H}\|u\|_{V}
$$

We will often use the short notation $B(u):=B(u, u)$. On the other hand, the nonlinear operator $P_{H}((u \cdot \nabla) v)$ is well defined whenever $u, v$ are such that $(u \cdot \nabla) v$ belongs to $L^{2}$. One can show that $P_{H}((u \cdot \nabla) v)$ can be linearly extended to $V \times V \longrightarrow V^{*}$, and actually coincides with previous $B(u, v)$.

It is known that the system (1.1) can be reformulated as follows:

$$
\left\{\begin{array}{l}
d u(t)=-A u(t) d t-B(u(t), u(t)) d t+F(u(t)) d t+\sum_{i=1}^{m} \sigma^{i}(u(t)) d W^{i}(t), \\
u(0)=h .
\end{array}\right.
$$


Let $F, \sigma^{i}, i=1, \cdots, m$ be measurable mappings from $H$ into $H$. We introduce the following condition:

(H.1) $F(\cdot), \sigma^{i}(\cdot): H \rightarrow H$ are globally Lipschitz maps, i.e., there exists a constant $C<\infty$ such that

$\left\|F\left(u_{1}\right)-F\left(u_{2}\right)\right\|_{H}^{2}+\sum_{i=1}^{m}\left\|\sigma^{i}\left(u_{1}\right)-\sigma^{i}\left(u_{2}\right)\right\|_{H}^{2} \leq C\left\|u_{1}-u_{2}\right\|_{H}^{2}, \quad \forall u_{1}, u_{2} \in H$.

Definition 2.1 A continuous $H$-valued $\left(\mathcal{F}_{t}\right)$-adapted process $u=(u(t))_{t \geq 0}$ is said to be a solution to equation (2.12) if for any $T>0, X \in L^{2}([0, T] \times$ $\Omega, d t \times P, V)$ and for any $t \geq 0$, the following equation holds in $V^{*}, P$-a.s.:

$u(t)=h-\int_{0}^{t} A u(s) d s-\int_{0}^{t} B(u(s)) d s+\int_{0}^{t} F(u(s)) d s+\sum_{i=1}^{m} \int_{0}^{t} \sigma^{i}(u(s)) d W^{i}(s)$.

Under the assumption (H.1) and $h \in H$, it is known that equation (2.12) admits a unique solution (see e.g. [4]).

\section{Approximations of SNSEs by pure jump type SNSEs}

For $\varepsilon>0$, let $\sigma^{i, \varepsilon}: H \times \mathbb{R}_{0} \rightarrow H$ be given measurable maps. Consider the following SNSE driven by pure jump noise:

$$
\begin{aligned}
u^{\varepsilon}(t)= & h-\int_{0}^{t} A u^{\varepsilon}(s) d s-\int_{0}^{t} B\left(u^{\varepsilon}(s)\right) d s+\int_{0}^{t} F\left(u^{\varepsilon}(s)\right) \\
& +\sum_{i=1}^{m} \int_{0}^{t} \int_{\mathbb{R}_{0}} \sigma^{i, \varepsilon}\left(u^{\varepsilon}(s-), z\right) \tilde{N}^{i}(d z d s) .
\end{aligned}
$$

We impose the following conditions on $\sigma^{i, \varepsilon}$.

(H.2) There exists constants $C>0$ and $\varepsilon_{0}>0$ such that

$$
\begin{gathered}
\|F(u)\|_{H}^{2}+\sup _{\varepsilon \leq \varepsilon_{0}} \sum_{i=1}^{m} \int_{\mathbb{R}_{0}}\left\|\sigma^{i, \varepsilon}(u, z)\right\|_{H}^{2} \nu^{i}(d z) \leq C\left(1+\|u\|_{H}^{2}\right), \\
\sup _{\varepsilon \leq \varepsilon_{0}} \sum_{i=1}^{m} \int_{\mathbb{R}_{0}}\left\|\sigma^{i, \varepsilon}(u, z)\right\|_{H}^{4} \nu^{i}(d z) \leq C\left(1+\|u\|_{H}^{4}\right),
\end{gathered}
$$


$\left\|F\left(u_{1}\right)-F\left(u_{2}\right)\right\|_{H}^{2}+\sup _{\varepsilon \leq \varepsilon_{0}} \sum_{i=1}^{m} \int_{\mathbb{R}_{0}}\left\|\sigma^{i, \varepsilon}\left(u_{1}, z\right)-\sigma^{i, \varepsilon}\left(u_{2}, z\right)\right\|_{H}^{2} \nu^{i}(d z) \leq C\left\|u_{1}-u_{2}\right\|_{H}^{2}$.

Denote by $D([0, T], H)$ the space of all càdlàg paths from $[0, T]$ into $H$ equipped with the Skorohod topology.

Definition 3.1 A $H$-valued $\left(\mathcal{F}_{t}\right)$-adapted process $u^{\varepsilon}=\left(u^{\varepsilon}(t)\right)_{t \geq 0}$ is said to be a solution to equation (3.1) if

(i) for any $T>0, u^{\varepsilon} \in D([0, T], H) \cap L^{2}([0, T] \times \Omega, d t \times P, V)$;

(ii) for every $t \geq 0$, (3.1) holds in $V^{*}, P$-a.s..

Under the assumption (H.2) and $h \in H$, it is known that for $\varepsilon \leq \varepsilon_{0}$, equation (3.1) admits a unique solution (see e.g. [4]).

Consider the following conditions.

(H.3) (i) For each $i \in\{1, \cdots, m\}, \forall M>0$,

$$
\sup _{\|u\|_{H} \leq M} \sup _{z \in \mathbb{R}_{0}}\left\|\sigma^{i, \varepsilon}(u, z)\right\|_{H} \stackrel{\varepsilon \rightarrow 0}{\longrightarrow} 0 .
$$

(ii) For each $i \in\{1, \cdots, m\}$ and each $k, j \in \mathbb{N}, u \in H$,

$$
\int_{\mathbb{R}_{0}}\left(\sigma^{i, \varepsilon}(u, z), e_{k}\right)\left(\sigma^{i, \varepsilon}(u, z), e_{j}\right) \nu^{i}(d z) \stackrel{\varepsilon \rightarrow 0}{\longrightarrow}\left(\sigma^{i}(u), e_{k}\right)\left(\sigma^{i}(u), e_{j}\right) .
$$

(H.4) For each $i \in\{1, \cdots, m\}$ and every $u \in H$,

$$
\int_{\mathbb{R}_{0}}\left\|\sigma^{i, \varepsilon}(u, z)\right\|_{H}^{2} \nu^{i}(d z) \stackrel{\varepsilon \rightarrow 0}{\longrightarrow}\left\|\sigma^{i}(u)\right\|_{H}^{2}
$$

Remark 3.2 For our considerations, the jumping measures $\nu^{i}, i=1, \cdots, m$ typically have infinite volume. Therefore, (i),(ii) in (H.3) and (H.4) do not imply each other.

(H.5) The maps $F, \sigma^{i, \varepsilon}$ take the space $V$ into itself and there exist constants $C>0$ and $\varepsilon_{0}>0$ such that

$$
\|F(u)\|_{V}^{2}+\sup _{\varepsilon \leq \varepsilon_{0}} \sum_{i=1}^{m} \int_{\mathbb{R}_{0}}\left\|\sigma^{i, \varepsilon}(u, z)\right\|_{V}^{2} \nu^{i}(d z) \leq C\left(1+\|u\|_{V}^{2}\right) .
$$




\subsection{Preliminary estimates}

We first prepare some preliminary results needed for the proofs of the main results. In the rest of the paper, for simplicity of the exposition, we let $m=1$ and omit the superscript $i$ of $\sigma^{i}, \widetilde{N}^{i}, \nu^{i}$. The case of $m>1$ does not cause extra difficulties.

Lemma 3.3 Assume (H.2) and $h \in H$, let $u^{\varepsilon}$ be the solution of equation (3.1), then we have

$$
\sup _{\varepsilon \leq \varepsilon_{0}}\left\{E \sup _{0 \leq t \leq T}\left\|u^{\varepsilon}(t)\right\|_{H}^{4}+E\left(\int_{0}^{T}\left\|u^{\varepsilon}(s)\right\|_{V}^{2} d s\right)^{2}\right\}<\infty .
$$

Remark 3.4 If we assume (H.1) and $h \in H$, then (3.9) also holds for the solution $u$ of equation (2.12).

Proof. By Itô's formula and (2.6), we have

$$
\begin{aligned}
\left\|u^{\varepsilon}(t)\right\|_{H}^{2}= & \|h\|_{H}^{2}-2 \int_{0}^{t}\left\langle A u^{\varepsilon}(s), u^{\varepsilon}(s)\right\rangle d s+2 \int_{0}^{t}\left(F\left(u^{\varepsilon}(s)\right), u^{\varepsilon}(s)\right) d s \\
& +M(t)+\int_{0}^{t} \int_{\mathbb{R}_{0}}\left\|\sigma^{\varepsilon}\left(u^{\varepsilon}(s-), z\right)\right\|_{H}^{2} \nu(d z) d s
\end{aligned}
$$

where

$$
M(t):=\int_{0}^{t} \int_{\mathbb{R}_{0}}\left(\left\|\sigma^{\varepsilon}\left(u^{\varepsilon}(s-), z\right)\right\|_{H}^{2}+2\left(\sigma^{\varepsilon}\left(u^{\varepsilon}(s-), z\right), u^{\varepsilon}(s-)\right)\right) \tilde{N}(d z d s) .
$$

Using Burkhölder's inequality and the assumption (H.2), we have

$$
\begin{aligned}
& E \sup _{0 \leq r \leq t}|M(r)|^{2} \\
\leq & C E \int_{0}^{t} \int_{\mathbb{R}_{0}}\left(\left\|\sigma^{\varepsilon}\left(u^{\varepsilon}(s-), z\right)\right\|_{H}^{2}+2\left(\sigma^{\varepsilon}\left(u^{\varepsilon}(s-), z\right), u^{\varepsilon}(s-)\right)\right)^{2} \nu(d z) d s \\
\leq & C E \int_{0}^{t}\left(1+\left\|u^{\varepsilon}(s)\right\|_{H}^{4}\right) d s .
\end{aligned}
$$

By (2.2), it follows from (3.10) that for $t \leq T$,

$$
\left\|u^{\varepsilon}(t)\right\|_{H}^{4}+\left(\int_{0}^{t}\left\|u^{\varepsilon}(s)\right\|_{V}^{2} d s\right)^{2} \leq C\|h\|_{H}^{4}+C \int_{0}^{t}\left(1+\left\|u^{\varepsilon}(s)\right\|_{H}^{4}\right) d s+C M(t)^{2} .
$$


Take superemum over the interval $[0, t]$ in (3.13), and use (3.12) to get

$E \sup _{0 \leq s \leq t}\left\|u^{\varepsilon}(s)\right\|_{H}^{4}+E\left(\int_{0}^{t}\left\|X_{s}^{\varepsilon}\right\|_{V}^{2} d s\right)^{2} \leq C\|h\|_{H}^{4}+C E \int_{0}^{t}\left(1+\left\|u^{\varepsilon}(s)\right\|_{H}^{4}\right) d s$.

Applying Gronwall's inequality completes the proof of the lemma.

Lemma 3.5 Assume (H.2), (H.5) and $h \in V$. For any constant $M>0$, define

$$
\tau_{M}^{\varepsilon}:=T \wedge \inf \left\{t \geq 0: \int_{0}^{t}\left\|u^{\varepsilon}(s)\right\|_{V}^{2} d s>M\right\} \wedge \inf \left\{t \geq 0:\left\|u^{\varepsilon}(t)\right\|_{H}^{2}>M\right\},
$$

where we set $\inf \{\emptyset\}=\infty$. Then we have

$$
\sup _{\varepsilon \leq \varepsilon_{0}}\left\{E \sup _{0 \leq t \leq \tau_{M}^{\varepsilon}}\left\|u^{\varepsilon}(t)\right\|_{V}^{2}+E\left(\int_{0}^{\tau_{M}^{\varepsilon}}\left\|u^{\varepsilon}(s)\right\|_{H^{2}}^{2} d s\right)^{2}\right\}<\infty .
$$

Proof. Through Galerkin approximations, it can be shown that for $\varepsilon \leq \varepsilon_{0}$, the solution $u^{\varepsilon} \in L^{\infty}([0, T], V) \cap L^{2}\left([0, T], H^{2}\right)$ with probability one (see e.g. Proposition 2.2 in [12]). Apply Itô's formula to $\left\|u^{\varepsilon}(t)\right\|_{V}^{2}$ to get

$$
\begin{aligned}
\left\|u^{\varepsilon}(t)\right\|_{V}^{2}= & \|h\|_{V}^{2}-2 \int_{0}^{t}\left\|A u^{\varepsilon}(s)\right\|_{H}^{2} d s-2 \int_{0}^{t}\left(B\left(u^{\varepsilon}(s)\right), A u^{\varepsilon}(s)\right) d s \\
& +2 \int_{0}^{t}\left(\left(F\left(u^{\varepsilon}(s)\right), u^{\varepsilon}(s)\right)\right) d s+M_{1}(t)+M_{2}(t)
\end{aligned}
$$

where

$$
\begin{aligned}
& M_{1}(t):=2 \int_{0}^{t} \int_{\mathbb{R}_{0}}\left(\left(\sigma^{\varepsilon}\left(u^{\varepsilon}(s-), z\right), u^{\varepsilon}(s-)\right)\right) \tilde{N}(d z d s), \\
& M_{2}(t):=\int_{0}^{t} \int_{\mathbb{R}_{0}}\left\|\sigma^{\varepsilon}\left(u^{\varepsilon}(s-), z\right)\right\|_{V}^{2} N(d z d s) .
\end{aligned}
$$

Use (2.8) and Young's inequality to obtain

$$
\left|\left(B\left(u^{\varepsilon}(s)\right), A u^{\varepsilon}(s)\right)\right| \leq C\left\|u^{\varepsilon}\right\|_{H^{2}}^{\frac{3}{2}}\left\|u^{\varepsilon}\right\|_{V}\left\|u^{\varepsilon}\right\|_{H}^{\frac{1}{2}} \leq\left\|u^{\varepsilon}\right\|_{H^{2}}^{2}+C\left\|u^{\varepsilon}\right\|_{V}^{4}\left\|u^{\varepsilon}\right\|_{H}^{2} .
$$

Therefore, by (3.20) and (H.5), we obtain

$$
\left\|u^{\varepsilon}(t)\right\|_{V}^{2}+\int_{0}^{t}\left\|u^{\varepsilon}(s)\right\|_{H^{2}}^{2} d s
$$


$\leq\|h\|_{V}^{2}+C \int_{0}^{t}\left\|u^{\varepsilon}(s)\right\|_{V}^{4}\left\|u^{\varepsilon}(s)\right\|_{H}^{2} d s+C \int_{0}^{t}\left(1+\left\|u^{\varepsilon}(s)\right\|_{V}^{2}\right) d s+M_{1}(t)+M_{2}(t)$.

Applying Gronwall's inequality yields that

$$
\begin{aligned}
& \left\|u^{\varepsilon}(t)\right\|_{V}^{2}+\int_{0}^{t}\left\|u^{\varepsilon}(s)\right\|_{H^{2}}^{2} d s \\
& \leq\left(C_{T}+\|h\|_{V}^{2}+\sup _{0 \leq t \leq \tau_{M}^{\varepsilon}}\left|M_{1}(t)\right|+M_{2}\left(\tau_{M}^{\varepsilon}\right)\right) \\
& \quad \times \exp \left(C_{T}+C \int_{0}^{t}\left\|u^{\varepsilon}(s)\right\|_{V}^{2}\left\|u^{\varepsilon}(s)\right\|_{H}^{2} d s\right), \quad t \in\left[0, \tau_{M}^{\varepsilon}\right] .
\end{aligned}
$$

Take superemum over the interval $\left[0, \tau_{M}^{\varepsilon}\right]$, remember the definition of $\tau_{M}^{\varepsilon}$ and take expectations to get

$$
\begin{aligned}
& E \sup _{0 \leq t \leq \tau_{M}^{\varepsilon}}\left\|u^{\varepsilon}(t)\right\|_{V}^{2}+E \int_{0}^{\tau_{M}^{\varepsilon}}\left\|u^{\varepsilon}(s)\right\|_{H^{2}}^{2} d s \\
\leq & \left(C_{T}+\|h\|_{V}^{2}+E \sup _{0 \leq t \leq \tau_{M}^{\varepsilon}}\left|M_{1}(t)\right|+E M_{2}\left(\tau_{M}^{\varepsilon}\right)\right) \exp \left(C_{T}+C M^{2}\right) .
\end{aligned}
$$

By Burkhölder's inequality, (H.5) and Young's inequality, we have for $\delta>0$,

$$
\begin{aligned}
E \sup _{0 \leq t \leq \tau_{M}^{\varepsilon}}\left|M_{1}(t)\right| & \leq 2 E\left[\int_{0}^{\tau_{M}^{\varepsilon}} \int_{\mathbb{R}_{0}}\left(\left(\sigma^{\varepsilon}\left(u^{\varepsilon}(s-), z\right), u^{\varepsilon}(s-)\right)\right)^{2} \nu(d z) d s\right]^{\frac{1}{2}} \\
& \leq 2 E\left[\int_{0}^{\tau_{M}^{\varepsilon}} C\left\|u^{\varepsilon}(s)\right\|_{V}^{2}\left(1+\left\|u^{\varepsilon}(s)\right\|_{V}^{2}\right) d s\right]^{\frac{1}{2}} \\
& \leq 2(C T+C M)^{\frac{1}{2}} E \sup _{0 \leq t \leq \tau_{M}^{\varepsilon}}\left\|u^{\varepsilon}(t)\right\|_{V} \\
& \leq \delta E \sup _{0 \leq t \leq \tau_{M}^{\varepsilon}}\left\|u^{\varepsilon}(t)\right\|_{V}^{2}+\frac{1}{\delta}(C T+C M)
\end{aligned}
$$

By (H.5) and (3.9), we have

$$
\begin{aligned}
E M_{2}\left(\tau_{M}^{\varepsilon}\right) & =E \int_{0}^{\tau_{M}^{\varepsilon}} \int_{\mathbb{R}_{0}}\left\|\sigma^{\varepsilon}\left(u^{\varepsilon}(s-), z\right)\right\|_{V}^{2} N(d z d s) \\
& =E \int_{0}^{\tau_{M}^{\varepsilon}} \int_{\mathbb{R}_{0}}\left\|\sigma^{\varepsilon}\left(u^{\varepsilon}(s-), z\right)\right\|_{V}^{2} \nu(d z) d s \\
& \leq E \int_{0}^{\tau_{M}^{\varepsilon}} C\left(1+\left\|u^{\varepsilon}(s)\right\|_{V}^{2}\right) d s \leq C<\infty .
\end{aligned}
$$


Combining (3.23), (3.24) and (3.25) and choosing sufficiently small $\delta$, we obtain

$$
E \sup _{0 \leq t \leq \tau_{M}^{\varepsilon}}\left\|u^{\varepsilon}(t)\right\|_{V}^{2}+E \int_{0}^{\tau_{M}^{\varepsilon}}\left\|u^{\varepsilon}(s)\right\|_{H^{2}}^{2} d s \leq C_{T, M}\|h\|_{V}^{2}+C_{T, M}
$$

completing the proof of (3.16).

Proposition 3.6 Assume (H.2), (H.5) and $h \in V$. Then the family $\left\{u^{\varepsilon}, \varepsilon \leq\right.$ $\left.\varepsilon_{0}\right\}$ is tight in the space $D([0, T], H)$.

Proof. Note that $V$ is compactly embedded into $H$. Thus, by Aldou's tightness criterion (see Theorem 1 in [1]), it suffices to show that:

(i) for any $0<\eta<1$, there exists $L_{\eta}>0$ such that

$$
\sup _{\varepsilon \leq \varepsilon_{0}} P\left(\sup _{0 \leq t \leq T}\left\|u^{\varepsilon}(t)\right\|_{V}>L_{\eta}\right)<\eta
$$

(ii) for any stopping time $0 \leq \zeta^{\varepsilon} \leq T$ with respect to the natural filtration generated by $\left\{u^{\varepsilon}(s), s \leq t\right\}$, and any $\eta>0$,

$$
\lim _{\delta \rightarrow 0} \sup _{\varepsilon \leq \varepsilon_{0}} P\left(\left\|u^{\varepsilon}\left(\zeta^{\varepsilon}+\delta\right)-u^{\varepsilon}\left(\zeta^{\varepsilon}\right)\right\|_{H}>\eta\right)=0,
$$

where we set $\zeta^{\varepsilon}+\delta:=T \wedge\left(\zeta^{\varepsilon}+\delta\right)$.

Note that (3.9) implies

$$
\begin{aligned}
& \sup _{\varepsilon \leq \varepsilon_{0}} P\left(\tau_{M}^{\varepsilon}<T\right) \\
\leq & \sup _{\varepsilon \leq \varepsilon_{0}} P\left(\int_{0}^{T}\left\|u^{\varepsilon}(s)\right\|_{V}^{2} d s>M\right)+\sup _{\varepsilon \leq \varepsilon_{0}} P\left(\sup _{0 \leq s \leq T}\left\|u^{\varepsilon}(s)\right\|_{H}^{2}>M\right) \\
\leq & \frac{1}{M} \sup _{\varepsilon \leq \varepsilon_{0}} E \int_{0}^{T}\left\|u^{\varepsilon}(s)\right\|_{V}^{2} d s+\frac{1}{M} \sup _{\varepsilon \leq \varepsilon_{0}} E \sup _{0 \leq t \leq T}\left\|u^{\varepsilon}(s)\right\|_{H}^{2} \\
\leq & \frac{C}{M} .
\end{aligned}
$$

For any $L>0$, by (3.29) and (3.16), we have

$$
\begin{aligned}
& \sup _{\varepsilon \leq \varepsilon_{0}} P\left(\sup _{0 \leq t \leq T}\left\|u^{\varepsilon}(t)\right\|_{V}>L\right) \\
\leq & \sup _{\varepsilon \leq \varepsilon_{0}} P\left(\sup _{0 \leq t \leq T}\left\|u^{\varepsilon}(t)\right\|_{V}>L, \tau_{M}^{\varepsilon}=T\right)+\sup _{\varepsilon \leq \varepsilon_{0}} P\left(\tau_{M}^{\varepsilon}<T\right)
\end{aligned}
$$




$$
\begin{aligned}
& \leq \sup _{\varepsilon \leq \varepsilon_{0}} P\left(\sup _{0 \leq t \leq \tau_{M}^{\varepsilon}}\left\|u^{\varepsilon}(t)\right\|_{V}>L\right)+\frac{C}{M} \\
& \leq \frac{1}{L^{2}} \sup _{\varepsilon \leq \varepsilon_{0}} E \sup _{0 \leq t \leq \tau_{M}^{\varepsilon}}\left\|u^{\varepsilon}(t)\right\|_{V}^{2}+\frac{C}{M} \\
& \leq \frac{C_{M}}{L^{2}}+\frac{C}{M} .
\end{aligned}
$$

Given any $\eta>0$, we can first take sufficiently large constant $M$, and then choose the constant $L$ so that the right hand side of (3.30) will be smaller than $\eta$. Hence (i) is satisfied.

Now, we come to verify (ii). For any $\eta>0$,

$$
\begin{aligned}
& \sup _{\varepsilon \leq \varepsilon_{0}} P\left(\left\|u^{\varepsilon}\left(\zeta^{\varepsilon}+\delta\right)-u^{\varepsilon}\left(\zeta^{\varepsilon}\right)\right\|_{H}>\eta\right) \\
& \leq \sup _{\varepsilon \leq \varepsilon_{0}} P\left(\left\|\int_{\zeta^{\varepsilon}}^{\zeta^{\varepsilon}+\delta} A u^{\varepsilon}(s) d s\right\|_{H}>\frac{\eta}{4}\right) \\
& \quad+\sup _{\varepsilon \leq \varepsilon_{0}} P\left(\left\|\int_{\zeta^{\varepsilon}}^{\zeta^{\varepsilon}+\delta} B\left(u^{\varepsilon}(s)\right) d s\right\|_{H}>\frac{\eta}{4}\right) \\
& \quad+\sup _{\varepsilon \leq \varepsilon_{0}} P\left(\left\|\int_{\zeta^{\varepsilon}}^{\zeta^{\varepsilon}+\delta} F\left(u^{\varepsilon}(s)\right) d s\right\|_{H}>\frac{\eta}{4}\right) \\
& \quad+\sup _{\varepsilon \leq \varepsilon_{0}} P\left(\left\|\int_{\zeta^{\varepsilon}}^{\zeta^{\varepsilon}+\delta} \int_{\mathbb{R}_{0}} \sigma^{\varepsilon}\left(u^{\varepsilon}(s-), z\right) \widetilde{N}(d z d s)\right\|_{H}>\frac{\eta}{4}\right) \\
& \quad:=I_{1}+I_{2}+I_{3}+I_{4} .
\end{aligned}
$$

By Hölder's inequality and Chebyshev's inequality, it follows from (3.16) and (3.29) that for $M>0$,

$$
\begin{aligned}
I_{1} & \leq \sup _{\varepsilon \leq \varepsilon_{0}} P\left(\delta \int_{\zeta^{\varepsilon}}^{\zeta^{\varepsilon}+\delta}\left\|A u^{\varepsilon}(s)\right\|_{H}^{2} d s>\frac{\eta^{2}}{16}\right) \\
& \leq \sup _{\varepsilon \leq \varepsilon_{0}} P\left(\delta \int_{\zeta^{\varepsilon}}^{\zeta^{\varepsilon}+\delta}\left\|A u^{\varepsilon}(s)\right\|_{H}^{2} d s>\frac{\eta^{2}}{16}, \tau_{M}^{\varepsilon}=T\right)+\sup _{\varepsilon \leq \varepsilon_{0}} P\left(\tau_{M}^{\varepsilon}<T\right) \\
& \leq \sup _{\varepsilon \leq \varepsilon_{0}} P\left(\delta \int_{0}^{\tau_{M}^{\varepsilon}}\left\|A u^{\varepsilon}(s)\right\|_{H}^{2} d s>\frac{\eta^{2}}{16}\right)+\frac{C}{M} \\
& \leq \frac{16}{\eta^{2}} \delta \sup _{\varepsilon \leq \varepsilon_{0}} E \int_{0}^{\tau_{M}^{\varepsilon}}\left\|A u^{\varepsilon}(s)\right\|_{H}^{2} d s+\frac{C}{M} \\
& \leq \frac{C_{M}}{\eta^{2}} \delta+\frac{C}{M} .
\end{aligned}
$$


By (2.8), we have $\left\|B\left(u^{\varepsilon}(s)\right)\right\|_{H} \leq C\left\|u^{\varepsilon}(s)\right\|_{H^{2}}^{\frac{1}{2}}\left\|u^{\varepsilon}(s)\right\|_{V}\left\|u^{\varepsilon}(s)\right\|_{H}^{\frac{1}{2}}$. Using (3.16) and (3.29), we have

$$
\begin{aligned}
I_{2} \leq & \sup _{\varepsilon \leq \varepsilon_{0}} P\left(\int_{\zeta^{\varepsilon}}^{\zeta^{\varepsilon}+\delta}\left\|B\left(u^{\varepsilon}(s)\right)\right\|_{H} d s>\frac{\eta}{4}\right) \\
\leq & \sup _{\varepsilon \leq \varepsilon_{0}} P\left(\int_{\zeta^{\varepsilon}}^{\zeta^{\varepsilon}+\delta}\left\|u^{\varepsilon}(s)\right\|_{H^{2}}^{\frac{1}{2}}\left\|u^{\varepsilon}(s)\right\|_{V}\left\|u^{\varepsilon}(s)\right\|_{H}^{\frac{1}{2}} d s>\frac{\eta}{4 C}\right) \\
\leq & \sup _{\varepsilon \leq \varepsilon_{0}} P\left(\int_{\zeta^{\varepsilon}}^{\zeta^{\varepsilon}+\delta}\left\|u^{\varepsilon}(s)\right\|_{H^{2}}^{\frac{1}{2}}\left\|u^{\varepsilon}(s)\right\|_{V}\left\|u^{\varepsilon}(s)\right\|_{H}^{\frac{1}{2}} d s>\frac{\eta}{4 C}, \tau_{M}^{\varepsilon}=T\right) \\
& +\sup _{\varepsilon \leq \varepsilon_{0}} P\left(\tau_{M}^{\varepsilon}<T\right) \\
\leq & \sup _{\varepsilon \leq \varepsilon_{0}} P\left(\int_{\zeta^{\varepsilon}}^{\left(\zeta^{\varepsilon}+\delta\right) \wedge \tau_{M}^{\varepsilon}}\left\|u^{\varepsilon}(s)\right\|_{H^{2}}^{\frac{1}{2}}\left\|u^{\varepsilon}(s)\right\|_{V}\left\|u^{\varepsilon}(s)\right\|_{H}^{\frac{1}{2}} d s>\frac{\eta}{4 C}\right)+\frac{C}{M} \\
\leq & \frac{4 C}{\eta} \sup _{\varepsilon \leq \varepsilon_{0}}\left[\left(E \int_{\zeta^{\varepsilon}}^{\left(\zeta^{\varepsilon}+\delta\right) \wedge \tau_{M}^{\varepsilon}}\left\|u^{\varepsilon}(s)\right\|_{H}^{2} d s\right)^{\frac{1}{4}}\left(E \int_{\zeta^{\varepsilon}}^{\left(\zeta^{\varepsilon}+\delta\right) \wedge \tau_{M}^{\varepsilon}}\left\|u^{\varepsilon}(s)\right\|_{H^{2}}^{2} d s\right)^{\frac{1}{4}}\right. \\
& \left.\times\left(E \int_{\zeta^{\varepsilon}}^{\left(\zeta^{\varepsilon}+\delta\right) \wedge \tau_{M}^{\varepsilon}}\left\|u^{\varepsilon}(s)\right\|_{V}^{2} d s\right)^{\frac{1}{2}}\right]+\frac{C}{M} \\
\leq & \frac{C_{M}}{\eta} \delta^{\frac{3}{4}} \sup _{\varepsilon \leq \varepsilon_{0}}\left(E \sup _{0 \leq s \leq T}\left\|u^{\varepsilon}(s)\right\|_{H}^{2}\right)^{\frac{1}{4}} \times \sup _{\varepsilon \leq \varepsilon_{0}}\left(E \int_{0}^{\tau_{M}^{\varepsilon}}\left\|u^{\varepsilon}(s)\right\|_{H^{2}}^{2} d s\right)^{\frac{1}{4}} \\
& \times \sup _{\varepsilon \leq \varepsilon_{0}}\left(E \sup _{0 \leq s \leq \tau_{M}^{\varepsilon}}\left\|u^{\varepsilon}(s)\right\|_{V}^{2}\right)^{\frac{1}{2}}+\frac{C}{M} \\
\leq & \frac{C_{M}}{\eta} \delta^{\frac{3}{4}}+\frac{C}{M} .
\end{aligned}
$$

On the other hand, by (H.2) and (3.9) we have

$$
\begin{aligned}
I_{3} & \leq \frac{4}{\eta} \sup _{\varepsilon \leq \varepsilon_{0}} E \int_{\zeta^{\varepsilon}}^{\zeta^{\varepsilon}+\delta}\left\|F\left(u^{\varepsilon}(s)\right)\right\|_{H} d s \\
& \leq \frac{4}{\eta} \sup _{\varepsilon \leq \varepsilon_{0}} E \int_{\zeta^{\varepsilon}}^{\zeta^{\varepsilon}+\delta} C\left(1+\left\|u^{\varepsilon}(s)\right\|_{H}\right) d s \\
& \leq \frac{C}{\eta} \delta\left(1+\sup _{\varepsilon \leq \varepsilon_{0}} E \sup _{0 \leq s \leq T}\left\|u^{\varepsilon}(s)\right\|_{H}\right) \\
& \leq \frac{C}{\eta} \delta .
\end{aligned}
$$

Similarly,

$$
I_{4} \leq \frac{16}{\eta^{2}} \sup _{\varepsilon \leq \varepsilon_{0}} E\left\|\int_{\zeta^{\varepsilon}}^{\zeta^{\varepsilon}+\delta} \int_{\mathbb{R}_{0}} \sigma^{\varepsilon}\left(u^{\varepsilon}(s-), z\right) \tilde{N}(d z d s)\right\|_{H}^{2}
$$




$$
\begin{aligned}
& \leq \frac{16}{\eta^{2}} \sup _{\varepsilon \leq \varepsilon_{0}} E \int_{\zeta^{\varepsilon}}^{\zeta^{\varepsilon}+\delta} \int_{\mathbb{R}_{0}}\left\|\sigma^{\varepsilon}\left(u^{\varepsilon}(s-), z\right)\right\|_{H}^{2} \nu(d z) d s \\
& \leq \frac{16}{\eta^{2}} \sup _{\varepsilon \leq \varepsilon_{0}} E \int_{\zeta^{\varepsilon}}^{\zeta^{\varepsilon}+\delta} C\left(1+\left\|u^{\varepsilon}(s)\right\|_{H}^{2}\right) d s \\
& \leq \frac{C}{\eta^{2}} \delta .
\end{aligned}
$$

Combine (3.32) - (3.35) together, first let $\delta \rightarrow 0$, then let $M \rightarrow \infty$ to obtain (3.28). Thus (ii) is verified, which completes the proof.

\subsection{The weak convergence}

Denote by $\mu_{\varepsilon}, \mu$ respectively the laws of $u^{\varepsilon}$ and $u$ on the spaces $D([0, T], H)$ and $C([0, T], H)$. We will establish the weak convergence by two stages. We first obtain the weak convergence in Theorem 3.7 under stronger conditions, and then we remove the extra assumptions and get the general convergence result in Theorem 3.8.

Theorem 3.7 Assume (H.1), (H.2), (H.3), (H.5) and $h \in V$. Then, for any $T>0, \mu_{\varepsilon}$ converges weakly to $\mu$, as $\varepsilon \rightarrow 0$, on the space $D([0, T], H)$ equipped with the Skorohod topology.

Proof. By Proposition 3.6, the family $\left\{\mu_{\varepsilon}, \varepsilon \leq \varepsilon_{0}\right\}$ is tight in $D([0, T], H)$. Let $\mu_{0}$ be the weak limit of any convergent subsequence $\left\{\mu_{\varepsilon_{n}}\right\}$. We will show that $\mu_{0}=\mu$. The rest of the proof is divided into three steps. In step 1 , we show that $\mu_{0}$ is supported on the space $C([0, T], H)$. In step 2 , we prove that $\mu_{0}$ is a solution of a martingale problem. In step 3 , we show that $\mu_{0}$ is the law of a weak solution of SNSE (2.12), hence complete the proof.

Step 1. For any $\eta>0, M>0$, we have

$$
\begin{aligned}
& P\left(\sup _{0<t \leq T}\left\|u^{\varepsilon}(t)-u^{\varepsilon}(t-)\right\|_{H}>\eta\right) \\
\leq & P\left(\sup _{0<t \leq T} \sup _{z \in \mathbb{R}_{0}}\left\|\sigma^{\varepsilon}\left(u^{\varepsilon}(t-), z\right)\right\|_{H}>\eta\right) \\
\leq & P\left(\sup _{0 \leq t \leq T} \sup _{z \in \mathbb{R}_{0}}\left\|\sigma^{\varepsilon}\left(u^{\varepsilon}(t), z\right)\right\|_{H}>\eta, \sup _{0 \leq t \leq T}\left\|u^{\varepsilon}(t)\right\| \leq M\right) \\
& +P\left(\sup _{0 \leq t \leq T}\left\|u^{\varepsilon}(t)\right\|>M\right) \\
\leq & P\left(\sup _{\|x\|_{H} \leq M} \sup _{z \in \mathbb{R}_{0}}\left\|\sigma^{\varepsilon}(x, z)\right\|_{H}>\eta\right)+\frac{1}{M^{2}} \sup _{\varepsilon \leq \varepsilon_{0}} E \sup _{0 \leq t \leq T}\left\|u^{\varepsilon}(t)\right\|_{H}^{2} .
\end{aligned}
$$


By (3.9) and (3.5), we first let $\varepsilon \rightarrow 0$ and then $M \rightarrow \infty$ to see that

$$
\sup _{0<t \leq T}\left\|u^{\varepsilon}(t)-u^{\varepsilon}(t-)\right\|_{H} \stackrel{\varepsilon \rightarrow 0}{\longrightarrow} 0 \quad \text { in probability. }
$$

Therefore, it follows from Theorem 13.4 in [3] that $\mu_{0}$ is supported on the space $C([0, T], H)$. As a consequence, the finite dimensional distributions of $\mu_{\varepsilon_{n}}$ converge to that of $\mu_{0}$.

Step 2. For $k, j \in \mathbb{N}$, let $f(x)=\left(x, e_{k}\right)\left(x, e_{j}\right), x \in H$. The gradient of $f$ (denoted by $\nabla f$ ) and the operator (denoted by $f^{\prime \prime}$ ) associated with the second derivatives of $f$ are respectively given by

$$
\begin{gathered}
\nabla f(x)=\left(x, e_{j}\right) e_{k}+\left(x, e_{k}\right) e_{j}, \\
f^{\prime \prime}(x)=e_{j} \otimes e_{k}+e_{k} \otimes e_{j} .
\end{gathered}
$$

Set

$$
\begin{aligned}
L^{\varepsilon} f(x):= & -(A \nabla f(x), x)-\langle B(x), \nabla f(x)\rangle+(F(x), \nabla f(x)) \\
& +\int_{\mathbb{R}_{0}}\left[f\left(x+\sigma^{\varepsilon}(x, z)\right)-f(x)-\left(\nabla f(x), \sigma^{\varepsilon}(x, z)\right)\right] \nu(d z), \quad(3.40) \\
L f(x):= & -(A \nabla f(x), x)-\langle B(x), \nabla f(x)\rangle+(F(x), \nabla f(x))+\frac{1}{2}\left(f^{\prime \prime}(x) \sigma(x), \sigma(x)\right) .
\end{aligned}
$$

By Itô's formula,

$$
\begin{aligned}
& f\left(u^{\varepsilon}(t)\right)-f(h)-\int_{0}^{t} L^{\varepsilon} f\left(u^{\varepsilon}(s)\right) d s \\
= & \int_{0}^{t} \int_{\mathbb{R}_{0}}\left[f\left(u^{\varepsilon}(s-)+\sigma^{\varepsilon}\left(u^{\varepsilon}(s-), z\right)\right)-f\left(u^{\varepsilon}(s-)\right)\right] \tilde{N}(d z d s)
\end{aligned}
$$

is a martingale. Denote by $X_{t}(\omega):=\omega(t), \omega \in D([0, T], H)$ the coordinate process on $D([0, T], H)$. By the above martingale property, for any $0 \leq s_{0}<$ $s_{1}<\ldots<s_{n} \leq s<t$ and $f_{0}, f_{1}, \ldots f_{n} \in C_{b}(H)$ (the collection of bounded continuous functions on $H$ ), it holds that

$$
E^{\mu_{\varepsilon}}\left[\left(f\left(X_{t}\right)-f\left(X_{s}\right)-\int_{s}^{t} L^{\varepsilon} f\left(X_{r}\right) d r\right) f_{0}\left(X_{s_{0}}\right) \ldots f_{n}\left(X_{s_{n}}\right)\right]=0 .
$$

Let

$$
G_{\varepsilon}(x):=\left|\int_{\mathbb{R}_{0}}\left(\sigma^{\varepsilon}(x, z), e_{k}\right)\left(\sigma^{\varepsilon}(x, z), e_{j}\right) \nu(d z)-\left(\sigma(x), e_{k}\right)\left(\sigma(x), e_{j}\right)\right|,
$$


$x \in H$. By (3.40) and (3.41), we have

$$
\left|L^{\varepsilon} f\left(X_{r}\right)-L f\left(X_{r}\right)\right|=G_{\varepsilon}\left(X_{r}\right) .
$$

We claim that

$$
\lim _{n \rightarrow \infty} E^{\mu_{\varepsilon_{n}}}\left[\int_{s}^{t}\left|L^{\varepsilon_{n}} f\left(X_{r}\right)-L f\left(X_{r}\right)\right| d r\right]=0 .
$$

Note that

$$
\begin{gathered}
E^{\mu_{\varepsilon_{n}}}\left[\int_{s}^{t}\left|L^{\varepsilon_{n}} f\left(X_{r}\right)-L f\left(X_{r}\right)\right| d r\right]=\int_{s}^{t} E G_{\varepsilon_{n}}\left(u^{\varepsilon_{n}}(r)\right) d r \\
\sup _{\varepsilon \leq \varepsilon_{0}} G_{\varepsilon}(x) \leq C\left(1+\|x\|_{H}^{2}\right) .
\end{gathered}
$$

By the dominated convergence theorem and (3.9), to prove (3.46), it suffices to prove that for every $r \in[0, T]$,

$$
\lim _{n \rightarrow \infty} E G_{\varepsilon_{n}}\left(u^{\varepsilon_{n}}(r)\right)=0 .
$$

Since the finite dimensional distributions of $\mu_{\varepsilon_{n}}$ converge weakly to that of $\mu_{0}$, by the Skorohod's representation theorem, in order not to introduce more notations, we can assume that $u^{\varepsilon_{n}}(r)$ converges almost surely to a $H$-valued random variable $u^{0}$. In view of (3.9), $\left\{\left\|u^{\varepsilon_{n}}(r)\right\|_{H}^{2}\right\}_{n \geq 1}$ is uniformly integrable, and therefore we can further deduce that $u^{0} \in L^{2}(\Omega, H)$ and

$$
\lim _{n \rightarrow \infty} E\left\|u^{\varepsilon_{n}}(r)-u^{0}\right\|_{H}^{2}=0
$$

By the dominated convergence theorem, it follows from (3.6) and (3.48) that

$$
\lim _{n \rightarrow \infty} E G_{\varepsilon_{n}}\left(u^{0}\right)=0 .
$$

Hence to prove (3.49), it suffices to prove

$$
\lim _{n \rightarrow \infty} E\left|G_{\varepsilon_{n}}\left(u^{\varepsilon_{n}}(r)\right)-G_{\varepsilon_{n}}\left(u^{0}\right)\right|=0 .
$$

We have

$$
\begin{aligned}
& E\left|G_{\varepsilon_{n}}\left(u^{\varepsilon_{n}}(r)\right)-G_{\varepsilon_{n}}\left(u^{0}\right)\right| \\
& \leq E \mid \int_{\mathbb{R}_{0}}\left(\sigma^{\varepsilon_{n}}\left(u^{\varepsilon_{n}}(r), z\right), e_{k}\right)\left(\sigma^{\varepsilon_{n}}\left(u^{\varepsilon_{n}}(r), z\right), e_{j}\right) \nu(d z) \\
& \quad-\int_{\mathbb{R}_{0}}\left(\sigma^{\varepsilon_{n}}\left(u^{0}, z\right), e_{k}\right)\left(\sigma^{\varepsilon_{n}}\left(u^{0}, z\right), e_{j}\right) \nu(d z) \mid
\end{aligned}
$$




$$
\begin{aligned}
& +E\left|\left(\sigma\left(u^{\varepsilon_{n}}(r)\right), e_{k}\right)\left(\sigma\left(u^{\varepsilon_{n}}(r)\right), e_{j}\right)-\left(\sigma\left(u^{0}\right), e_{k}\right)\left(\sigma\left(u^{0}\right), e_{j}\right)\right| \\
:= & I_{1}+I_{2} .
\end{aligned}
$$

In view of (3.2) and (3.4), we have

$$
\begin{aligned}
I_{1} \leq & E \int_{\mathbb{R}_{0}}\left|\left(\sigma^{\varepsilon_{n}}\left(u^{\varepsilon_{n}}(r), z\right), e_{k}\right)\left(\sigma^{\varepsilon_{n}}\left(u^{\varepsilon_{n}}(r), z\right)-\sigma^{\varepsilon_{n}}\left(u^{0}, z\right), e_{j}\right)\right| \nu(d z) \\
& +E \int_{\mathbb{R}_{0}}\left|\left(\sigma^{\varepsilon_{n}}\left(u^{\varepsilon_{n}}(r), z\right)-\sigma^{\varepsilon_{n}}\left(u^{0}, z\right), e_{k}\right)\left(\sigma^{\varepsilon_{n}}\left(u^{0}, z\right), e_{j}\right)\right| \nu(d z) \\
\leq & {\left[E \int_{\mathbb{R}_{0}}\left\|\sigma^{\varepsilon_{n}}\left(u^{\varepsilon_{n}}(r), z\right)\right\|_{H}^{2} \nu(d z)\right]^{\frac{1}{2}}\left[E \int_{\mathbb{R}_{0}}\left\|\sigma^{\varepsilon_{n}}\left(u^{\varepsilon_{n}}(r), z\right)-\sigma^{\varepsilon_{n}}\left(u^{0}, z\right)\right\|_{H}^{2} \nu(d z)\right]^{\frac{1}{2}} } \\
& +\left[E \int_{\mathbb{R}_{0}}\left\|\sigma^{\varepsilon_{n}}\left(u^{0}, z\right)\right\|_{H}^{2} \nu(d z)\right]^{\frac{1}{2}}\left[E \int_{\mathbb{R}_{0}}\left\|\sigma^{\varepsilon_{n}}\left(u^{\varepsilon_{n}}(r), z\right)-\sigma^{\varepsilon_{n}}\left(u^{0}, z\right)\right\|_{H}^{2} \nu(d z)\right]^{\frac{1}{2}} \\
\leq & C\left[\left(1+E\left\|u^{0}\right\|_{H}^{2}\right)^{\frac{1}{2}}+\sup _{\varepsilon_{n}}\left(1+E\left\|u^{\varepsilon_{n}}(r)\right\|_{H}^{2}\right)^{\frac{1}{2}}\right]\left(E\left\|u^{\varepsilon_{n}}(r)-u^{0}\right\|_{H}^{2}\right)^{\frac{1}{2}} .
\end{aligned}
$$

This yields that $I_{1} \rightarrow 0$ taking into account (3.9) and (3.50). A similar argument leads to $I_{2} \rightarrow 0$. Therefore, (3.52) holds. Hence the claim (3.46) is proved.

Next we prove that

$$
M_{k, j}(t):=f\left(X_{t}\right)-f(h)-\int_{0}^{t} L f\left(X_{r}\right) d r
$$

is a martingale under $\mu_{0}$. This is equivalent to proving that

$$
E^{\mu_{0}}\left[\left(f\left(X_{t}\right)-f\left(X_{s}\right)-\int_{s}^{t} L f\left(X_{r}\right) d r\right) f_{0}\left(X_{s_{0}}\right) \ldots f_{n}\left(X_{s_{n}}\right)\right]=0 .
$$

Since the finite dimensional distributions of $\mu_{\varepsilon_{n}}$ converge to that of $\mu_{0}$, noticing that $\|f(x)\|_{H} \leq\|x\|_{H}^{2}$ and (3.9), it follows from Theorem 1.6.8 in [7] that

$$
E^{\mu_{0}}\left[f\left(X_{t}\right) f_{0}\left(X_{s_{0}}\right) \cdots f_{n}\left(X_{s_{n}}\right)\right]=\lim _{n \rightarrow \infty} E^{\mu_{\varepsilon_{n}}}\left[f\left(X_{t}\right) f_{0}\left(X_{s_{0}}\right) \ldots f_{n}\left(X_{s_{n}}\right)\right] .
$$

In view of (2.4), we have

$$
\left|\left\langle B(x, x), e_{k}\right\rangle\right|=\left|\left\langle B\left(x, e_{k}\right), x\right\rangle\right| \leq C\|x\|_{H}^{2}\left\|\nabla e_{k}\right\|_{L^{\infty}} \leq C\left\|e_{k}\right\|_{H^{3}}\|x\|_{H}^{2} .
$$

Thus, $L f(x)$ is a continuous function on $H$ and

$$
|L f(x)| \leq C\left(1+\|x\|_{H}^{3}\right) .
$$


Therefore, for the same reason as (3.57), we have for every $r \in[s, t]$,

$$
E^{\mu_{0}}\left[\left(L f\left(X_{r}\right)\right) f_{0}\left(X_{s_{0}}\right) \ldots f_{n}\left(X_{s_{n}}\right)\right]=\lim _{n \rightarrow \infty} E^{\mu_{\varepsilon_{n}}}\left[\left(L f\left(X_{r}\right)\right) f_{0}\left(X_{s_{0}}\right) \ldots f_{n}\left(X_{s_{n}}\right)\right] .
$$

By the Fubini theorem and the dominate convergence theorem, we obtain

$$
\begin{aligned}
& E^{\mu_{0}}\left[\left(\int_{s}^{t} L f\left(X_{r}\right) d r\right) f_{0}\left(X_{s_{0}}\right) \ldots f_{n}\left(X_{s_{n}}\right)\right] \\
= & \lim _{n \rightarrow \infty} E^{\mu_{\varepsilon_{n}}}\left[\left(\int_{s}^{t} L f\left(X_{r}\right) d r\right) f_{0}\left(X_{s_{0}}\right) \ldots f_{n}\left(X_{s_{n}}\right)\right] .
\end{aligned}
$$

Using (3.57), (3.61), (3.46) and (3.43), we have

$$
\begin{aligned}
& E^{\mu_{0}}\left[\left(f\left(X_{t}\right)-f\left(X_{s}\right)-\int_{s}^{t} L f\left(X_{r}\right) d r\right) f_{0}\left(X_{s_{0}}\right) \ldots f_{n}\left(X_{s_{n}}\right)\right] \\
= & \lim _{n \rightarrow \infty} E^{\mu_{\varepsilon_{n}}}\left[\left(f\left(X_{t}\right)-f\left(X_{s}\right)-\int_{s}^{t} L f\left(X_{r}\right) d r\right) f_{0}\left(X_{s_{0}}\right) \ldots f_{n}\left(X_{s_{n}}\right)\right] \\
= & \lim _{n \rightarrow \infty} E^{\mu_{\varepsilon_{n}}}\left[\left(f\left(X_{t}\right)-f\left(X_{s}\right)-\int_{s}^{t} L^{\varepsilon_{n}} f\left(X_{r}\right) d r\right) f_{0}\left(X_{s_{0}}\right) \ldots f_{n}\left(X_{s_{n}}\right)\right] \\
= & 0 .
\end{aligned}
$$

Hence $M_{k, j}(t)$ in $(3.55)$ is a martingale under $\mu_{0}$.

For $k \in \mathbb{N}$, let $g(x)=\left(x, e_{k}\right), x \in H$. By a similar argument, we can show that

$$
\begin{aligned}
& M_{k}(t):=g\left(X_{t}\right)-g(h)-\int_{0}^{t} L g\left(X_{r}\right) d r \\
= & \left(X_{t}, e_{k}\right)-\left(h, e_{k}\right)+\int_{0}^{t}\left(A e_{k}, X_{s}\right) d s+\int_{0}^{t}\left\langle B\left(X_{s}\right), e_{k}\right\rangle d s-\int_{0}^{t}\left(F\left(X_{s}\right), e_{k}\right) d s
\end{aligned}
$$

is a martingale under $\mu_{0}$.

Step 3. (3.55) and (3.63) together with Itô's formula yield that

$$
<M_{k}, M_{j}>(t)=\int_{0}^{t}\left(\sigma\left(X_{s}\right), e_{k}\right)\left(\sigma\left(X_{s}\right), e_{j}\right) d s,
$$

where $<M_{k}, M_{j}>$ stands for the sharp bracket of the two martingales. Now by Theorem 18.12 in [11], there exists a probability space $\left(\Omega^{\prime}, \mathcal{F}^{\prime}, P^{\prime}\right)$ with a filtration $\mathcal{F}_{t}^{\prime}$ such that on the standard extension

$$
\left(\Omega \times \Omega^{\prime}, \mathcal{F} \times \mathcal{F}^{\prime}, \mathcal{F}_{t} \times \mathcal{F}_{t}^{\prime}, \mu_{0} \times P^{\prime}\right)
$$


of $\left(\Omega, \mathcal{F}, \mathcal{F}_{t}, P\right)$ there exists a one-dimensional Brownian motion $W(t), t \geq 0$ such that

$$
M_{k}(t)=\int_{0}^{t}\left(\sigma\left(X_{s}\right), e_{k}\right) d W(s)
$$

namely,

$$
\begin{aligned}
\left(X_{t}, e_{k}\right)-\left(h, e_{k}\right)= & -\int_{0}^{t}\left(A e_{k}, X_{s}\right) d s-\int_{0}^{t}\left\langle B\left(X_{s}\right), e_{k}\right\rangle d s \\
& +\int_{0}^{t}\left(F\left(X_{s}\right), e_{k}\right) d s+\int_{0}^{t}\left(\sigma\left(X_{s}\right), e_{k}\right) d W(s)
\end{aligned}
$$

for every $k \geq 1$. Thus, under $\mu_{0},\left\{X_{t}, t \geq 0\right\}$ is a solution to SNSE (2.12). By the uniqueness of the SNSE, we conclude that $\mu_{0}=\mu$ completing the proof of the theorem.

In the next theorem, we will remove the restrictions placed on the coefficients and the initial value $h$.

Theorem 3.8 Assume (H.1), (H.2), (H.3), (H.4) and $h \in H$. Then, for any $T>0, \mu_{\varepsilon}$ converges weakly to $\mu$, as $\varepsilon \rightarrow 0$, on the space $D([0, T], H)$ equipped with the Skorohod topology.

Proof. For each $n \in \mathbb{N}$, let $h^{n}, F_{n}(u), \sigma_{n}(u), \sigma_{n}^{\varepsilon}(u, z)$ denote the corresponding orthogonal projections of $h, F(u), \sigma(u), \sigma^{\varepsilon}(u, z)$ into the $n$-dimensional space $\operatorname{span}\left\{e_{1}, \cdots, e_{n}\right\}$. Then, for each $n \in \mathbb{N},\left\{\sigma_{n}^{\varepsilon}\right\}_{\varepsilon \leq \varepsilon_{0}}$ and $F_{n}$ satisfy (H.2) - (H.5). Moreover, there is a constant $C$ independent of $n$ such that for every $u, u_{1}, u_{2} \in H$,

$$
\begin{aligned}
\sup _{n \in \mathbb{N}} \| & F_{n}(u)\left\|_{H}^{2}+\sup _{n \in \mathbb{N}}\right\| \sigma_{n}(u)\left\|_{H}^{2}+\sup _{n \in \mathbb{N}, \varepsilon \leq \varepsilon_{0}} \int_{\mathbb{R}_{0}}\right\| \sigma_{n}^{\varepsilon}(u, z) \|_{H}^{2} \nu(d z) \leq C\left(1+\|u\|_{H}^{2}\right), \\
& \sup _{n \in \mathbb{N}}\left\|F_{n}\left(u_{1}\right)-F_{n}\left(u_{2}\right)\right\|_{H}^{2}+\sup _{n \in \mathbb{N}}\left\|\sigma_{n}\left(u_{1}\right)-\sigma_{n}\left(u_{2}\right)\right\|_{H}^{2} \\
& +\sup _{n \in \mathbb{N}, \varepsilon \leq \varepsilon_{0}} \int_{\mathbb{R}_{0}}\left\|\sigma_{n}^{\varepsilon}\left(u_{1}, z\right)-\sigma_{n}^{\varepsilon}\left(u_{2}, z\right)\right\|_{H}^{2} \nu(d z) \leq C\left\|u_{1}-u_{2}\right\|_{H}^{2}
\end{aligned}
$$

Let $u^{n, \varepsilon}, u^{n}$ be the solutions of the SNSEs:

$$
\begin{aligned}
u^{n, \varepsilon}(t)= & h^{n}-\int_{0}^{t} A u^{n, \varepsilon}(s) d s-\int_{0}^{t} B\left(u^{n, \varepsilon}(s)\right) d s+\int_{0}^{t} F_{n}\left(u^{n, \varepsilon}(s)\right) d s \\
& +\int_{0}^{t} \int_{\mathbb{R}_{0}} \sigma_{n}^{\varepsilon}\left(u^{n, \varepsilon}(s-), z\right) \tilde{N}(d z d s),
\end{aligned}
$$




$$
\begin{aligned}
u^{n}(t)= & h^{n}-\int_{0}^{t} A u^{n}(s) d s-\int_{0}^{t} B\left(u^{n}(s)\right) d s+\int_{0}^{t} F_{n}\left(u^{n}(s)\right) d s \\
& +\int_{0}^{t} \sigma_{n}\left(u^{n}(s)\right) d W(s) .
\end{aligned}
$$

By Theorem 3.7, we have for each $n \in \mathbb{N}$,

$$
u^{n, \varepsilon} \stackrel{\varepsilon \rightarrow 0}{\longrightarrow} u^{n} \quad \text { in distribution on the space } D([0, T], H) .
$$

Moreover, as the proof of (3.9), using (3.67) we can show that

$$
\begin{array}{r}
\sup _{n \in \mathbb{N}, \varepsilon \leq \varepsilon_{0}}\left\{E \sup _{0 \leq t \leq T}\left\|u^{n, \varepsilon}(t)\right\|_{H}^{4}+E\left(\int_{0}^{T}\left\|u^{n, \varepsilon}(s)\right\|_{V}^{2} d s\right)^{2}\right\}<\infty, \\
\sup _{n \in \mathbb{N}}\left\{E \sup _{0 \leq t \leq T}\left\|u^{n}(t)\right\|_{H}^{4}+E\left(\int_{0}^{T}\left\|u^{n}(s)\right\|_{V}^{2} d s\right)^{2}\right\}<\infty .
\end{array}
$$

We claim that for any $\delta>0$,

$$
\begin{gathered}
\lim _{n \rightarrow \infty} P\left(\sup _{0 \leq t \leq T}\left\|u^{n}(t)-u(t)\right\|_{H}>\delta\right)=0, \\
\lim _{n \rightarrow \infty} \lim _{\varepsilon \rightarrow 0} P\left(\sup _{0 \leq t \leq T}\left\|u^{n, \varepsilon}(t)-u^{\varepsilon}(t)\right\|_{H}>\delta\right)=0 .
\end{gathered}
$$

Because of similarity, we only prove (3.75) here. Applying Itô's formula, we have

$$
\begin{aligned}
& e^{-\gamma \int_{0}^{t}\left\|u^{\varepsilon}(\rho)\right\|_{V}^{2} d \rho}\left\|u^{n, \varepsilon}(t)-u^{\varepsilon}(t)\right\|_{H}^{2} \\
= & \left\|h^{n}-h\right\|_{H}^{2}-\gamma \int_{0}^{t} e^{-\gamma \int_{0}^{s}\left\|u^{\varepsilon}(\rho)\right\|_{V}^{2} d \rho}\left\|u^{n, \varepsilon}(s)-u^{\varepsilon}(s)\right\|_{H}^{2}\left\|u^{\varepsilon}(s)\right\|_{V}^{2} d s \\
& -2 \int_{0}^{t} e^{-\gamma \int_{0}^{s}\left\|u^{\varepsilon}(\rho)\right\|_{V}^{2} d \rho}\left\langle A\left(u^{n, \varepsilon}(s)-u^{\varepsilon}(s)\right), u^{n, \varepsilon}(s)-u^{\varepsilon}(s)\right\rangle d s \\
& -2 \int_{0}^{t} e^{-\gamma \int_{0}^{s}\left\|u^{\varepsilon}(\rho)\right\|_{V}^{2} d \rho}\left\langle B\left(u^{n, \varepsilon}(s)\right)-B\left(u^{\varepsilon}(s)\right), u^{n, \varepsilon}(s)-u^{\varepsilon}(s)\right\rangle d s \\
& +2 \int_{0}^{t} e^{-\gamma \int_{0}^{s}\left\|u^{\varepsilon}(\rho)\right\|_{V}^{2} d \rho}\left(F_{n}\left(u^{n, \varepsilon}(s)\right)-F\left(u^{\varepsilon}(s)\right), u^{n, \varepsilon}(s)-u^{\varepsilon}(s)\right) d s \\
& +2 \int_{0}^{t} \int_{\mathbb{R}_{0}} e^{-\gamma \int_{0}^{s}\left\|u^{\varepsilon}(\rho)\right\|_{V}^{2} d \rho} \times \\
& \left(\sigma_{n}^{\varepsilon}\left(u^{n, \varepsilon}(s-), z\right)-\sigma^{\varepsilon}\left(u^{\varepsilon}(s-), z\right), u^{n, \varepsilon}(s-)-u^{\varepsilon}(s-)\right) \widetilde{N}(d z d s) \\
& +\int_{0}^{t} \int_{\mathbb{R}_{0}} e^{-\gamma \int_{0}^{s}\left\|u^{\varepsilon}(\rho)\right\|_{V}^{2} d \rho}\left\|\sigma_{n}^{\varepsilon}\left(u^{n, \varepsilon}(s-), z\right)-\sigma^{\varepsilon}\left(u^{\varepsilon}(s-), z\right)\right\|_{H}^{2} N(d z d s)
\end{aligned}
$$




$$
:=\sum_{k=1}^{7} I_{k}^{n, \varepsilon}(t) .
$$

By (2.6) and (2.7) we have

$$
\begin{aligned}
& 2\left|\left\langle B\left(u^{n, \varepsilon}(s)\right)-B\left(u^{\varepsilon}(s)\right), u^{n, \varepsilon}(s)-u^{\varepsilon}(s)\right\rangle\right|=2\left|\left\langle B\left(u^{n, \varepsilon}(s)-u^{\varepsilon}(s)\right), u^{\varepsilon}(s)\right\rangle\right| \\
\leq & 4\left\|u^{n, \varepsilon}(s)-u^{\varepsilon}(s)\right\|_{V}\left\|u^{n, \varepsilon}(s)-u^{\varepsilon}(s)\right\|_{H}\left\|u^{\varepsilon}(s)\right\|_{V} \\
\leq & \left\|u^{n, \varepsilon}(s)-u^{\varepsilon}(s)\right\|_{V}^{2}+4\left\|u^{\varepsilon}(s)\right\|_{V}^{2}\left\|u^{n, \varepsilon}(s)-u^{\varepsilon}(s)\right\|_{H}^{2} .
\end{aligned}
$$

Therefore, by (2.2) and (3.77) we obtain that

$$
\begin{aligned}
\sum_{k=2}^{4} I_{k}^{n, \varepsilon}(t) \leq \int_{0}^{t} e^{-\gamma \int_{0}^{s}\left\|u^{\varepsilon}(\rho)\right\|_{V}^{2} d \rho}\left[-\left\|u^{n, \varepsilon}(s)-u^{\varepsilon}(s)\right\|_{V}^{2}\right. \\
\left.\quad+(4-\gamma)\left\|u^{\varepsilon}(s)\right\|_{V}^{2}\left\|u^{n, \varepsilon}(s)-u^{\varepsilon}(s)\right\|_{H}^{2}\right] d s \\
\leq-\int_{0}^{t} e^{-\gamma \int_{0}^{s}\left\|u^{\varepsilon}(\rho)\right\|_{V}^{2} d \rho}\left\|u^{n, \varepsilon}(s)-u^{\varepsilon}(s)\right\|_{V}^{2} d s
\end{aligned}
$$

if we take $\gamma \geq 4$. Using the Lipschitz continuity of $F$, we have

$$
\begin{aligned}
& E \sup _{0 \leq s \leq t}\left|I_{5}^{n, \varepsilon}(s)\right| \\
\leq & E \int_{0}^{t} e^{-\gamma \int_{0}^{s}\left\|u^{\varepsilon}(\rho)\right\|_{V}^{2} d \rho}\left\|u^{n, \varepsilon}(s)-u^{\varepsilon}(s)\right\|_{H}^{2} d s \\
& +E \int_{0}^{t} e^{-\gamma \int_{0}^{s}\left\|u^{\varepsilon}(\rho)\right\|_{V}^{2} d \rho}\left\|F_{n}\left(u^{n, \varepsilon}(s)\right)-F\left(u^{\varepsilon}(s)\right)\right\|_{H}^{2} d s \\
\leq & E \int_{0}^{t} e^{-\gamma \int_{0}^{s}\left\|u^{\varepsilon}(\rho)\right\|_{V}^{2} d \rho}\left\|u^{n, \varepsilon}(s)-u^{\varepsilon}(s)\right\|_{H}^{2} d s \\
& +2 E \int_{0}^{t} e^{-\gamma \int_{0}^{s}\left\|u^{\varepsilon}(\rho)\right\|_{V}^{2} d \rho}\left\|F_{n}\left(u^{n, \varepsilon}(s)\right)-F\left(u^{n, \varepsilon}(s)\right)\right\|_{H}^{2} d s \\
& +2 E \int_{0}^{t} e^{-\gamma \int_{0}^{s}\left\|u^{\varepsilon}(\rho)\right\|_{V}^{2} d \rho}\left\|F\left(u^{n, \varepsilon}(s)\right)-F\left(u^{\varepsilon}(s)\right)\right\|_{H}^{2} d s \\
\leq & C E \int_{0}^{t} e^{-\gamma \int_{0}^{s}\left\|u^{\varepsilon}(\rho)\right\|_{V}^{2} d \rho}\left\|u^{n, \varepsilon}(s)-u^{\varepsilon}(s)\right\|_{H}^{2} d s \\
& +2 E \int_{0}^{t} e^{-\gamma \int_{0}^{s}\left\|u^{\varepsilon}(\rho)\right\|_{V}^{2} d \rho}\left\|F_{n}\left(u^{n, \varepsilon}(s)\right)-F\left(u^{n, \varepsilon}(s)\right)\right\|_{H}^{2} d s,
\end{aligned}
$$

By Burkhölder's inequality, we get

$$
E \sup _{0 \leq s \leq t}\left|I_{6}^{n, \varepsilon}(s)\right|
$$




$$
\begin{aligned}
& \leq 2 E\left[\int_{0}^{t} \int_{\mathbb{R}_{0}} e^{-2 \gamma \int_{0}^{s}\left\|u^{\varepsilon}(\rho)\right\|_{V}^{2} d \rho}\left\|\sigma_{n}^{\varepsilon}\left(u^{n, \varepsilon}(s), z\right)-\sigma^{\varepsilon}\left(u^{\varepsilon}(s), z\right)\right\|_{H}^{2} \times\right. \\
& \left.\left\|u^{n, \varepsilon}(s)-u^{\varepsilon}(s)\right\|_{H}^{2} \nu(d z) d s\right]^{\frac{1}{2}} \\
& \leq 2 E\left[\sup _{0 \leq s \leq t} e^{-\frac{\gamma}{2} \int_{0}^{s}\left\|u^{\varepsilon}(\rho)\right\|_{V}^{2} d \rho}\left\|u^{n, \varepsilon}(s)-u^{\varepsilon}(s)\right\|_{H} \times\right. \\
& \left.\quad\left(\int_{0}^{t} \int_{\mathbb{R}_{0}} e^{-\gamma \int_{0}^{s}\left\|u^{\varepsilon}(\rho)\right\|_{V}^{2} d \rho}\left\|\sigma_{n}^{\varepsilon}\left(u^{n, \varepsilon}(s), z\right)-\sigma^{\varepsilon}\left(u^{\varepsilon}(s), z\right)\right\|_{H}^{2} \nu(d z) d s\right)^{\frac{1}{2}}\right] \\
& \leq \frac{1}{2} E \sup _{0 \leq s \leq t} e^{-\gamma \int_{0}^{s}\left\|u^{\varepsilon}(\rho)\right\|_{V}^{2} d \rho}\left\|u^{n, \varepsilon}(s)-u^{\varepsilon}(s)\right\|_{H}^{2} \\
& +2 E \int_{0}^{t} \int_{\mathbb{R}_{0}} e^{-\gamma \int_{0}^{s}\left\|u^{\varepsilon}(\rho)\right\|_{V}^{2} d \rho}\left\|\sigma_{n}^{\varepsilon}\left(u^{n, \varepsilon}(s), z\right)-\sigma^{\varepsilon}\left(u^{\varepsilon}(s), z\right)\right\|_{H}^{2} \nu(d z) d s \\
& \leq \frac{1}{2} E \sup _{0 \leq s \leq t} e^{-\gamma \int_{0}^{s}\left\|u^{\varepsilon}(\rho)\right\|_{V}^{2} d \rho}\left\|u^{n, \varepsilon}(s)-u^{\varepsilon}(s)\right\|_{H}^{2} \\
& +C E \int_{0}^{t} e^{-\gamma \int_{0}^{s}\left\|u^{\varepsilon}(\rho)\right\|_{V}^{2} d \rho}\left\|u^{n, \varepsilon}(s)-u^{\varepsilon}(s)\right\|_{H}^{2} d s \\
& \quad+4 E \int_{0}^{t} \int_{\mathbb{R}_{0}} e^{-\gamma \int_{0}^{s}\left\|u^{\varepsilon}(\rho)\right\|_{V}^{2} d \rho}\left\|\sigma_{n}^{\varepsilon}\left(u^{n, \varepsilon}(s), z\right)-\sigma^{\varepsilon}\left(u^{n, \varepsilon}(s), z\right)\right\|_{H}^{2} \nu(d z) d s
\end{aligned}
$$

where the uniform Lipschitz constant of $\sigma^{\varepsilon}$ has been used. Similar to (3.79), we have

$$
\begin{aligned}
& E \sup _{0 \leq s \leq t}\left|I_{7}^{n, \varepsilon}(s)\right| \\
= & E \int_{0}^{t} \int_{\mathbb{R}_{0}} e^{-\gamma \int_{0}^{s}\left\|u^{\varepsilon}(\rho)\right\|_{V}^{2} d \rho}\left\|\sigma_{n}^{\varepsilon}\left(u^{n, \varepsilon}(s), z\right)-\sigma^{\varepsilon}\left(u^{\varepsilon}(s), z\right)\right\|_{H}^{2} \nu(d z) d s \\
\leq & C E \int_{0}^{t} e^{-\gamma \int_{0}^{s}\left\|u^{\varepsilon}(\rho)\right\|_{V}^{2} d \rho}\left\|u^{n, \varepsilon}(s)-u^{\varepsilon}(s)\right\|_{H}^{2} d s \\
& +2 E \int_{0}^{t} \int_{\mathbb{R}_{0}} e^{-\gamma \int_{0}^{s}\left\|u^{\varepsilon}(\rho)\right\|_{V}^{2} d \rho}\left\|\sigma_{n}^{\varepsilon}\left(u^{n, \varepsilon}(s), z\right)-\sigma^{\varepsilon}\left(u^{n, \varepsilon}(s), z\right)\right\|_{H}^{2} \nu(d z) d s .
\end{aligned}
$$

Combining (3.76), (3.78)-(3.81) together yields that for $t \leq T$,

$$
\begin{aligned}
& E \sup _{0 \leq s \leq t} e^{-\gamma \int_{0}^{s}\left\|u^{\varepsilon}(\rho)\right\|_{V}^{2} d \rho}\left\|u^{n, \varepsilon}(s)-u^{\varepsilon}(s)\right\|_{H}^{2} \\
& +2 E \int_{0}^{t} e^{-\gamma \int_{0}^{s}\left\|u^{\varepsilon}(\rho)\right\|_{V}^{2} d \rho}\left\|u^{n, \varepsilon}(s)-u^{\varepsilon}(s)\right\|_{V}^{2} d s
\end{aligned}
$$




$$
\begin{aligned}
\leq & 2\left\|h^{n}-h\right\|_{H}^{2}+C E \int_{0}^{t} e^{-\gamma \int_{0}^{s}\left\|u^{\varepsilon}(\rho)\right\|_{V}^{2} d \rho}\left\|u^{n, \varepsilon}(s)-u^{\varepsilon}(s)\right\|_{H}^{2} d s \\
& +4 E \int_{0}^{t} e^{-\gamma \int_{0}^{s}\left\|u^{\varepsilon}(\rho)\right\|_{V}^{2} d \rho}\left\|F_{n}\left(u^{n, \varepsilon}(s)\right)-F\left(u^{n, \varepsilon}(s)\right)\right\|_{H}^{2} d s \\
& +12 E \int_{0}^{t} e^{-\gamma \int_{0}^{s}\left\|u^{\varepsilon}(\rho)\right\|_{V}^{2} d \rho} \int_{\mathbb{R}_{0}}\left\|\sigma_{n}^{\varepsilon}\left(u^{n, \varepsilon}(s), z\right)-\sigma^{\varepsilon}\left(u^{n, \varepsilon}(s), z\right)\right\|_{H}^{2} \nu(d z) d s .
\end{aligned}
$$

Applying the Gronwall's inequality we obtain for $t \in[0, T]$,

$$
\begin{aligned}
& E \sup _{0 \leq s \leq t} e^{-\gamma \int_{0}^{s}\left\|u^{\varepsilon}(\rho)\right\|_{V}^{2} d \rho}\left\|u^{n, \varepsilon}(s)-u^{\varepsilon}(s)\right\|_{H}^{2} \\
& +E \int_{0}^{t} e^{-\gamma \int_{0}^{s}\left\|u^{\varepsilon}(\rho)\right\|_{V}^{2} d \rho}\left\|u^{n, \varepsilon}(s)-u^{\varepsilon}(s)\right\|_{V}^{2} d s \\
& \leq C \times\left[\left\|h^{n}-h\right\|_{H}^{2}+E \int_{0}^{t}\left\|F_{n}\left(u^{n, \varepsilon}(s)\right)-F\left(u^{n, \varepsilon}(s)\right)\right\|_{H}^{2} d s\right. \\
& \left.\quad+E \int_{0}^{t} \int_{\mathbb{R}_{0}}\left\|\sigma_{n}^{\varepsilon}\left(u^{n, \varepsilon}(s), z\right)-\sigma^{\varepsilon}\left(u^{n, \varepsilon}(s), z\right)\right\|_{H}^{2} \nu(d z) d s\right] .
\end{aligned}
$$

We claim that

$$
\begin{gathered}
\lim _{n \rightarrow \infty} \lim _{\varepsilon \rightarrow 0} E \int_{0}^{T} \int_{\mathbb{R}_{0}}\left\|\sigma_{n}^{\varepsilon}\left(u^{n, \varepsilon}(s), z\right)-\sigma^{\varepsilon}\left(u^{n, \varepsilon}(s), z\right)\right\|_{H}^{2} \nu(d z) d s=0, \\
\lim _{n \rightarrow \infty} \lim _{\varepsilon \rightarrow 0} E \int_{0}^{T}\left\|F_{n}\left(u^{n, \varepsilon}(s)\right)-F\left(u^{n, \varepsilon}(s)\right)\right\|_{H}^{2} d s=0 .
\end{gathered}
$$

Suppose the above claims are proved. Then we conclude from (3.83) that

$$
\lim _{n \rightarrow \infty} \lim _{\varepsilon \rightarrow 0} E \sup _{0 \leq s \leq T} e^{-\gamma \int_{0}^{s}\left\|u^{\varepsilon}(\rho)\right\|_{V}^{2} d \rho}\left\|u^{n, \varepsilon}(s)-u^{\varepsilon}(s)\right\|_{H}^{2}=0 .
$$

Let us only prove (3.84). The proof of (3.85) is similar and simpler. Let

$$
G_{n}^{\varepsilon}(x):=\int_{\mathbb{R}_{0}}\left\|\sigma_{n}^{\varepsilon}(x, z)-\sigma^{\varepsilon}(x, z)\right\|_{H}^{2} \nu(d z), \quad x \in H .
$$

Note that

$$
\sup _{n \in \mathbb{N}, \varepsilon \leq \varepsilon_{0}} G_{n}^{\varepsilon}(x) \leq C\left(1+\|x\|_{H}^{2}\right) .
$$

By (3.72) and the dominated convergence theorem, to prove (3.84), it suffices to show that for each $s \in[0, T]$,

$$
\lim _{n \rightarrow \infty} \lim _{\varepsilon \rightarrow 0} E G_{n}^{\varepsilon}\left(u^{n, \varepsilon}(s)\right)=0 .
$$


Obviously, (3.89) will follow if the following three equalities are proved.

$$
\begin{aligned}
\lim _{\varepsilon \rightarrow 0} E G_{n}^{\varepsilon}\left(u^{n, \varepsilon}(s)\right) & =\lim _{\varepsilon \rightarrow 0} E G_{n}^{\varepsilon}\left(u^{n}(s)\right), \quad \forall n \in \mathbb{N}, \\
\lim _{n \rightarrow \infty} \lim _{\varepsilon \rightarrow 0} E G_{n}^{\varepsilon}\left(u^{n}(s)\right) & =\lim _{n \rightarrow \infty} \lim _{\varepsilon \rightarrow 0} E G_{n}^{\varepsilon}(u(s)), \\
\lim _{n \rightarrow \infty} \lim _{\varepsilon \rightarrow 0} E G_{n}^{\varepsilon}(u(s)) & =0 .
\end{aligned}
$$

We first prove (3.90). Since $u^{n}$ is a continuous process, due to (3.71), we see that for each $n \in \mathbb{N}, s \in[0, T]$,

$$
u^{n, \varepsilon}(s) \stackrel{\varepsilon \rightarrow 0}{\longrightarrow} u^{n}(s) \quad \text { in distribution. }
$$

Therefore, to prove (3.90), we can use the Skorohod's representation theorem to assume that $\left\|u^{n, \varepsilon}(s)-u^{n}\right\|_{H} \rightarrow 0$ almost surely as $\varepsilon \rightarrow 0$. In view of (3.72), $\left\{\left\|u^{n, \varepsilon}(s)\right\|_{H}^{2}\right\}_{\varepsilon \leq \varepsilon_{0}}$ is uniformly integrable, and therefore, we can further deduce that

$$
\lim _{\varepsilon \rightarrow 0} E\left\|u^{n, \varepsilon}(s)-u^{n}(s)\right\|_{H}^{2}=0 .
$$

On the other hand,

$$
\begin{aligned}
& E\left|G_{n}^{\varepsilon}\left(u^{n, \varepsilon}(s)\right)-G_{n}^{\varepsilon}\left(u^{n}(s)\right)\right| \\
\leq & E \int_{\mathbb{R}_{0}}\left|\left\|\sigma_{n}^{\varepsilon}\left(u^{n, \varepsilon}(s), z\right)-\sigma^{\varepsilon}\left(u^{n, \varepsilon}(s), z\right)\right\|_{H}^{2}-\left\|\sigma_{n}^{\varepsilon}\left(u^{n}(s), z\right)-\sigma^{\varepsilon}\left(u^{n}(s), z\right)\right\|_{H}^{2}\right| \nu(d z) \\
\leq & E \int_{\mathbb{R}_{0}}\left(\left\|\sigma_{n}^{\varepsilon}\left(u^{n, \varepsilon}(s), z\right)-\sigma_{n}^{\varepsilon}\left(u^{n}(s), z\right)\right\|_{H}+\left\|\sigma^{\varepsilon}\left(u^{n, \varepsilon}(s), z\right)-\sigma^{\varepsilon}\left(u^{n}(s), z\right)\right\|_{H}\right) \\
& \times\left(\left\|\sigma_{n}^{\varepsilon}\left(u^{n, \varepsilon}(s), z\right)-\sigma^{\varepsilon}\left(u^{n, \varepsilon}(s), z\right)\right\|_{H}+\left\|\sigma_{n}^{\varepsilon}\left(u^{n}(s), z\right)-\sigma^{\varepsilon}\left(u^{n}(s), z\right)\right\|_{H}\right) \nu(d z) \\
\leq & {\left[2 E \int _ { \mathbb { R } _ { 0 } } \left(\left\|\sigma_{n}^{\varepsilon}\left(u^{n, \varepsilon}(s), z\right)-\sigma_{n}^{\varepsilon}\left(u^{n}(s), z\right)\right\|_{H}^{2}\right.\right.} \\
& \left.\left.\quad+\left\|\sigma^{\varepsilon}\left(u^{n, \varepsilon}(s), z\right)-\sigma^{\varepsilon}\left(u^{n}(s), z\right)\right\|_{H}^{2}\right) \nu(d z)\right]^{\frac{1}{2}} \\
& \quad\left[4 E \int _ { \mathbb { R } _ { 0 } } \left(\left\|\sigma_{n}^{\varepsilon}\left(u^{n, \varepsilon}(s), z\right)\right\|_{H}^{2}+\left\|\sigma^{\varepsilon}\left(u^{n, \varepsilon}(s), z\right)\right\|_{H}^{2}+\left\|\sigma_{n}^{\varepsilon}\left(u^{n}(s), z\right)\right\|_{H}^{2}\right.\right. \\
:= & I_{1}^{\varepsilon} \times I_{2}^{\varepsilon} .
\end{aligned}
$$

By (3.67), (3.2), (3.72) and (3.73), we deduce that

$$
\sup _{\varepsilon \leq \varepsilon_{0}}\left|I_{2}^{\varepsilon}\right|^{2} \leq C \sup _{n \in \mathbb{N}, \varepsilon \leq \varepsilon_{0}} E\left(1+\left\|u^{n, \varepsilon}(s)\right\|_{H}^{2}+\left\|u^{n}(s)\right\|_{H}^{2}\right)<\infty .
$$


(3.4), (3.68) and (3.94) imply

$$
\left|I_{1}^{\varepsilon}\right|^{2} \leq C E\left\|u^{n, \varepsilon}(s)-u^{n}(s)\right\|_{H}^{2} \stackrel{\varepsilon \rightarrow 0}{\longrightarrow} 0 .
$$

Therefore, (3.90) follows from (3.95), (3.96) and (3.97). In view of (3.74), a similar argument leads to

$$
\lim _{n \rightarrow \infty} \sup _{\varepsilon \leq \varepsilon_{0}} E\left|G_{n}^{\varepsilon}\left(u^{n}(s)\right)-G_{n}^{\varepsilon}(u(s))\right|=0 .
$$

Hence (3.91) holds. Note that (H.4) and the (ii) of (H.3) imply

$$
\begin{aligned}
& \lim _{n \rightarrow \infty} \lim _{\varepsilon \rightarrow 0} \int_{\mathbb{R}_{0}}\left\|\sigma_{n}^{\varepsilon}(x, z)-\sigma^{\varepsilon}(x, z)\right\|_{H}^{2} \nu(d z) \\
= & \lim _{n \rightarrow \infty} \lim _{\varepsilon \rightarrow 0}\left[\int_{\mathbb{R}_{0}}\left\|\sigma^{\varepsilon}(x, z)\right\|_{H}^{2} \nu(d z)-\int_{\mathbb{R}_{0}}\left\|\sigma_{n}^{\varepsilon}(x, z)\right\|_{H}^{2} \nu(d z)\right] \\
= & \|\sigma(x)\|_{H}^{2}-\lim _{n \rightarrow \infty}\left\|\sigma_{n}(x)\right\|_{H}^{2}=0, \quad \forall x \in H .
\end{aligned}
$$

Therefore, (3.92) immediately follows from (3.99) and (3.88) by the dominated convergence theorem. Thus, (3.84) is proved, and so is (3.86).

Next, we proceed with the proof of (3.75). For any given $\delta_{1}>0$, in view of (3.9), we can choose a positive constant $M_{1}$ such that

$$
\begin{aligned}
& \sup _{n \in \mathbb{N}, \varepsilon \leq \varepsilon_{0}} P\left(\sup _{0 \leq t \leq T}\left\|u^{n, \varepsilon}(t)-u^{\varepsilon}(t)\right\|_{H}>\delta, \int_{0}^{T}\left\|u^{\varepsilon}(s)\right\|_{V}^{2} d s>M_{1}\right) \\
\leq & \sup _{n \in \mathbb{N}, \varepsilon \leq \varepsilon_{0}} P\left(\int_{0}^{T}\left\|u^{\varepsilon}(s)\right\|_{V}^{2} d s>M_{1}\right) \leq \delta_{1}
\end{aligned}
$$

On the other hand, by (3.86), we have

$$
\begin{aligned}
& \lim _{n \rightarrow \infty} \lim _{\varepsilon \rightarrow 0} P\left(\sup _{0 \leq t \leq T}\left\|u^{n, \varepsilon}(t)-u^{\varepsilon}(t)\right\|_{H}>\delta, \int_{0}^{T}\left\|u^{\varepsilon}(s)\right\|_{V}^{2} d s \leq M_{1}\right) \\
\leq & \lim _{n \rightarrow \infty} \lim _{\varepsilon \rightarrow 0} P\left(\sup _{0 \leq s \leq T} e^{-\gamma \int_{0}^{s}\left\|u^{\varepsilon}(\rho)\right\|_{V}^{2} d \rho}\left\|u^{n, \varepsilon}(s)-u^{\varepsilon}(s)\right\|_{H}^{2} \geq e^{-\gamma M_{1}} \delta^{2}\right) \\
\leq & e^{\gamma M_{1}} \frac{1}{\delta^{2}} \lim _{n \rightarrow \infty} \lim _{\varepsilon \rightarrow 0} E \sup _{0 \leq s \leq T} e^{-\gamma \int_{0}^{s}\left\|u^{\varepsilon}(\rho)\right\|_{V}^{2} d \rho}\left\|u^{n, \varepsilon}(s)-u^{\varepsilon}(s)\right\|_{H}^{2}=0 .
\end{aligned}
$$

Combining (3.100) and (3.101) together yields

$$
\lim _{n \rightarrow \infty} \lim _{\varepsilon \rightarrow 0} P\left(\sup _{0 \leq t \leq T}\left\|u^{n, \varepsilon}(t)-u^{\varepsilon}(t)\right\|_{H}>\delta\right) \leq \delta_{1} .
$$


Since $\delta_{1}$ is arbitrary, (3.75) is proved.

Finally we prove that $\mu^{\varepsilon}$ converges weakly to $\mu$. Let $\mu_{n}^{\varepsilon}, \mu_{n}$ denote respectively the laws of $u^{n, \varepsilon}$ and $u^{n}$ on $S:=D([0, T], H)$. Let $G$ be any given bounded, uniformly continuous function on $S$. For any $n \geq 1$, we write

$$
\begin{aligned}
& \int_{S} G(w) \mu^{\varepsilon}(d w)-\int_{S} G(w) \mu(d w) \\
= & \int_{S} G(w) \mu^{\varepsilon}(d w)-\int_{S} G(w) \mu_{n}^{\varepsilon}(d w)+\int_{S} G(w) \mu_{n}^{\varepsilon}(d w)-\int_{S} G(w) \mu_{n}(d w) \\
& +\int_{S} G(w) \mu_{n}(d w)-\int_{S} G(w) \mu(d w) \\
= & E\left[G\left(u^{\varepsilon}\right)-G\left(u^{n, \varepsilon}\right)\right]+\left(\int_{S} G(w) \mu_{n}^{\varepsilon}(d w)-\int_{S} G(w) \mu_{n}(d w)\right) \\
& +E\left[G\left(u^{n}\right)-G(u)\right] .
\end{aligned}
$$

Give any $\delta>0$. Since $G$ is uniformly continuous, there exists $\delta_{1}>0$ such that

$$
\left|E\left[G\left(u^{\varepsilon}\right)-G\left(u^{n, \varepsilon}\right) ; \sup _{0 \leq s \leq T}\left\|u^{n, \varepsilon}(s)-u^{\varepsilon}(s)\right\|_{H} \leq \delta_{1}\right]\right| \leq \frac{\delta}{4}
$$

for all $n \geq 1, \varepsilon>0$. In view of (3.75) and (3.74), there exists $n_{1}$ and then $\varepsilon_{n_{1}}$ such that

$$
\begin{aligned}
& \sup _{\varepsilon \leq \varepsilon_{n_{1}}}\left|E\left[G\left(u^{\varepsilon}\right)-G\left(u^{n_{1}, \varepsilon}\right) ; \sup _{0 \leq s \leq T}\left\|u^{n_{1}, \varepsilon}(s)-u^{\varepsilon}(s)\right\|_{H}>\delta_{1}\right]\right| \\
\leq & C \sup _{\varepsilon \leq \varepsilon_{n_{1}}} P\left(\sup _{0 \leq s \leq T}\left\|u^{n_{1}, \varepsilon}(s)-u^{\varepsilon}(s)\right\|_{H}>\delta_{1}\right) \leq \frac{\delta}{4},
\end{aligned}
$$

and

$$
\left|E\left[G\left(u^{n_{1}}\right)-G(u)\right]\right| \leq \frac{\delta}{4}
$$

On the other hand, by (3.71), there exists $\varepsilon_{1}>0$ such that for $\varepsilon \leq \varepsilon_{1}$,

$$
\left|\int_{S} G(w) \mu_{n_{1}}^{\varepsilon}(d w)-\int_{S} G(w) \mu_{n_{1}}(d w)\right| \leq \frac{\delta}{4}
$$

Putting (3.103)-(3.107) together, we obtain that for $\varepsilon \leq \min \left\{\varepsilon_{n_{1}}, \varepsilon_{1}\right\}$,

$$
\left|\int_{S} G(w) \mu^{\varepsilon}(d w)-\int_{S} G(w) \mu(d w)\right| \leq \delta .
$$


Since $\delta>0$ is arbitrarily small, we deduce that

$$
\lim _{\varepsilon \rightarrow 0} \int_{S} G(w) \mu^{\varepsilon}(d w)=\int_{S} G(w) \mu(d w)
$$

completing the proof of the Theorem.

\section{Examples}

In this section, we give some examples of $\left\{\sigma^{\varepsilon}\right\}$ which satisfy the Hypotheses in Section 3.

Proposition 4.1 For each $\varepsilon>0$, let

$$
\sigma^{\varepsilon}(u, z)=\sigma\left(\theta_{\varepsilon}(z) u\right) h_{\varepsilon}(z), \quad u \in H, z \in \mathbb{R}_{0},
$$

where $\left\{\theta_{\varepsilon}(\cdot)\right\},\left\{h_{\varepsilon}(\cdot)\right\}$ are two families of real-valued functions on $\mathbb{R}_{0}$. Assume that $\left\{\theta_{\varepsilon}\right\}$ satisfies

$$
\sup _{z \in \mathbb{R}_{0}}\left|\theta_{\varepsilon}(z)-1\right| \stackrel{\varepsilon \rightarrow 0}{\longrightarrow} 0
$$

and $\left\{h_{\varepsilon}\right\}$ satisfies

$$
\begin{array}{r}
\int_{\mathbb{R}_{0}}\left|h_{\varepsilon}(z)\right|^{2} \nu(d z) \stackrel{\varepsilon \rightarrow 0}{\longrightarrow} 1, \\
\sup _{z \in \mathbb{R}_{0}}\left|h_{\varepsilon}(z)\right| \stackrel{\varepsilon \rightarrow 0}{\longrightarrow} 0 .
\end{array}
$$

Then $\left\{\sigma^{\varepsilon}\right\}$ satisfis (H.2)-(H.4).

Proof. By (4.2), there exists a constant $\varepsilon_{1}$ such that

$$
\sup _{\varepsilon \leq \varepsilon_{1}} \sup _{z \in \mathbb{R}_{0}}\left|\theta_{\varepsilon}(z)\right| \leq 2
$$

By (4.3), there exists a constant $\varepsilon_{2}$ such that

$$
\sup _{\varepsilon \leq \varepsilon_{2}} \int_{\mathbb{R}_{0}}\left|h_{\varepsilon}(z)\right|^{2} \nu(d z) \leq 2 .
$$

By (4.4), there exists a constant $\varepsilon_{3}$ such that

$$
\sup _{\varepsilon \leq \varepsilon_{3}} \sup _{z \in \mathbb{R}_{0}}\left|h_{\varepsilon}(z)\right| \leq 1 \text {. }
$$


Let $\varepsilon_{0}=\min \left\{\varepsilon_{1}, \varepsilon_{2}, \varepsilon_{3}\right\}$ and assume $\varepsilon \leq \varepsilon_{0}$ in the following calculation. The linear growth condition for $\sigma$ together with (4.5) and (4.4) yield

$$
\begin{aligned}
\sup _{\|x\|_{H} \leq M} \sup _{z \in \mathbb{R}_{0}}\left\|\sigma^{\varepsilon}(x, z)\right\|_{H} & =\sup _{\|x\|_{H} \leq M} \sup _{z \in \mathbb{R}_{0}}\left\|\sigma\left(\theta_{\varepsilon}(z) x\right)\right\|_{H}\left|h_{\varepsilon}(z)\right| \\
& \leq \sup _{\|x\|_{H} \leq M} \sup _{z \in \mathbb{R}_{0}} C\left(1+\left|\theta_{\varepsilon}(z)\right|\|x\|_{H}\right) \sup _{z \in \mathbb{R}_{0}}\left|h_{\varepsilon}(z)\right| \\
& \leq C(1+2 M) \sup _{z \in \mathbb{R}_{0}}\left|h_{\varepsilon}(z)\right| \stackrel{\varepsilon \rightarrow 0}{\longrightarrow} 0
\end{aligned}
$$

Thus, (i) of (H.3) is satisfied. By the Lipschitz condition of $\sigma$, we have

$$
\begin{aligned}
& \left.\left|\int_{\mathbb{R}_{0}}\left(\sigma\left(\theta_{\varepsilon}(z) x\right), e_{k}\right)\left(\sigma\left(\theta_{\varepsilon}(z) x\right), e_{j}\right)\right| h_{\varepsilon}(z)\right|^{2} \nu(d z) \\
& -\int_{\mathbb{R}_{0}}\left(\sigma(x), e_{k}\right)\left(\sigma(x), e_{j}\right)\left|h_{\varepsilon}(z)\right|^{2} \nu(d z) \mid \\
& \leq \int_{\mathbb{R}_{0}}\left|\left(\sigma\left(\theta_{\varepsilon}(z) x\right)-\sigma(x), e_{k}\right)\left(\sigma\left(\theta_{\varepsilon}(z) x\right), e_{j}\right)\right|\left|h_{\varepsilon}(z)\right|^{2} \nu(d z) \\
& +\int_{\mathbb{R}_{0}}\left|\left(\sigma(x), e_{k}\right)\left(\sigma\left(\theta_{\varepsilon}(z) x\right)-\sigma(x), e_{j}\right)\right|\left|h_{\varepsilon}(z)\right|^{2} \nu(d z) \\
& \leq\left[\int_{\mathbb{R}_{0}}\left\|\sigma\left(\theta_{\varepsilon}(z) x\right)-\sigma(x)\right\|_{H}^{2}\left|h_{\varepsilon}(z)\right|^{2} \nu(d z)\right]^{\frac{1}{2}} \\
& \times\left[\int_{\mathbb{R}_{0}}\left\|\sigma\left(\theta_{\varepsilon}(z) x\right)\right\|_{H}^{2}\left|h_{\varepsilon}(z)\right|^{2} \nu(d z)\right]^{\frac{1}{2}} \\
& +\left[\int_{\mathbb{R}_{0}}\left\|\sigma\left(\theta_{\varepsilon}(z) x\right)-\sigma(x)\right\|_{H}^{2}\left|h_{\varepsilon}(z)\right|^{2} \nu(d z)\right]^{\frac{1}{2}} \\
& \times\left[\int_{\mathbb{R}_{0}}\|\sigma(x)\|_{H}^{2}\left|h_{\varepsilon}(z)\right|^{2} \nu(d z)\right]^{\frac{1}{2}} \\
& \leq C \sup _{z \in \mathbb{R}_{0}}\left|\theta_{\varepsilon}(z)-1\right|\|x\|_{H}\left[\int_{\mathbb{R}_{0}}\left|h_{\varepsilon}(z)\right|^{2} \nu(d z)\right]^{\frac{1}{2}} \times\left\{\left[\int_{\mathbb{R}_{0}}\left(1+\left|\theta_{\varepsilon}(z)\right|^{2}\|x\|_{H}^{2}\right)\right.\right. \\
& \left.\left.\times\left|h_{\varepsilon}(z)\right|^{2} \nu(d z)\right]^{\frac{1}{2}}+\left[\int_{\mathbb{R}_{0}}\left(1+\|x\|_{H}^{2}\right)\left|h_{\varepsilon}(z)\right|^{2} \nu(d z)\right]^{\frac{1}{2}}\right\} \\
& \stackrel{\varepsilon \rightarrow 0}{\longrightarrow} 0
\end{aligned}
$$

where we have used (4.5), (4.6) and (4.2). On the other hand, (4.3) gives

$$
\int_{\mathbb{R}_{0}}\left(\sigma(x), e_{k}\right)\left(\sigma(x), e_{j}\right)\left|h_{\varepsilon}(z)\right|^{2} \nu(d z) \stackrel{\varepsilon \rightarrow 0}{\longrightarrow}\left(\sigma(x), e_{k}\right)\left(\sigma(x), e_{j}\right) .
$$

Combining (4.9) with (4.10), (ii) of (H.3) is obtained. (H.2) and (H.4) can be similarly verified, we omit the details. 
Example 4.2 Here we give some example of $\theta_{\varepsilon}$ and $h_{\varepsilon}$.

$$
\theta_{\varepsilon}(z)=1,1+\varepsilon \cos z, 1-\frac{\varepsilon}{\sqrt{2 \pi}} \mathrm{e}^{-\frac{\varepsilon^{2} z^{2}}{2}}, \cdots
$$

The following examples of $h_{\varepsilon}$ satisfy the conditions in Proposition 4.1.

(i)

$$
h_{\varepsilon}(z)=\frac{1}{\sqrt{\nu(\{\varepsilon \leq|z| \leq 1\})}} \mathbf{1}_{\{\varepsilon \leq|z| \leq 1\}}
$$

where the characteristic measure $\nu$ satisfies

$$
\nu(\{\varepsilon \leq|z| \leq 1\}) \stackrel{\varepsilon \rightarrow 0}{\longrightarrow} \infty, \quad \text { i.e. } \quad \nu\left(\mathbb{R}_{0}\right)=\infty .
$$

(ii)

$$
h_{\varepsilon}(z)=\frac{z}{\sqrt{\int_{1 \leq|z| \leq \frac{1}{\varepsilon}}|z|^{2} \nu(d z)}} \mathbf{1}_{\left\{1 \leq|z| \leq \frac{1}{\varepsilon}\right\}},
$$

where the characteristic measure $\nu$ satisfies

$$
\varepsilon^{2} \int_{1 \leq|z| \leq \frac{1}{\varepsilon}}|z|^{2} \nu(d z) \stackrel{\varepsilon \rightarrow 0}{\longrightarrow} \infty .
$$

(iii)

$$
h_{\varepsilon}(z)=\frac{z}{\sqrt{\int_{0<|z| \leq \varepsilon}|z|^{2} \nu(d z)}} \mathbf{1}_{\{0<|z| \leq \varepsilon\}}
$$

where the characteristic measure $\nu$ satisfies

$$
\frac{1}{\varepsilon^{2}} \int_{0<|z| \leq \varepsilon}|z|^{2} \nu(d z) \stackrel{\varepsilon \rightarrow 0}{\longrightarrow} \infty .
$$

For example, if $\nu_{\alpha}(d z)=\frac{1}{|z|^{1+\alpha}} d z$, which is the characteristic measure of symmetric $\alpha$-stable processes, then for each $\alpha \in(0,2)$, $\nu_{\alpha}$ satisfies (4.13), (4.15) and (4.17).

Acknowledgements This work is partly supported by National Natural Science Foundation of China (No.11671372, No.11431014, No.11401557). 


\section{References}

[1] D. Aldous. Stopping times and tightness. Ann. Probability, 6(2):335340, 1978.

[2] A. Bensoussan and R. Temam. Équations stochastiques du type NavierStokes. J. Funct. Anal., 13:195-222, 1973.

[3] P. Billingsley. Convergence of probability measures. Wiley Series in Probability and Statistics: Probability and Statistics. John Wiley \& Sons, Inc., New York, second edition, 1999. A Wiley-Interscience Publication.

[4] Z. Brzeźniak, W. Liu, and J. Zhu. Strong solutions for SPDE with locally monotone coefficients driven by Lévy noise. Nonlinear Anal. Real World Appl., 17:283-310, 2014.

[5] G. Di Nunno and T. Zhang. Approximations of stochastic partial differential equations. Ann. Appl. Probab., 26(3):1443-1466, 2016.

[6] Z. Dong, J. Xiong, J. Zhai, and T. Zhang. A moderate deviation principle for 2-D stochastic Navier-Stokes equations driven by multiplicative Lévy noises. J. Funct. Anal., 272(1):227-254, 2017.

[7] R. Durrett. Probability: theory and examples, volume 31 of Cambridge Series in Statistical and Probabilistic Mathematics. Cambridge University Press, Cambridge, fourth edition, 2010.

[8] F. Flandoli. Dissipativity and invariant measures for stochastic NavierStokes equations. NoDEA Nonlinear Differential Equations Appl., 1(4):403-423, 1994.

[9] M. Hairer and J. C. Mattingly. Ergodicity of the 2D Navier-Stokes equations with degenerate stochastic forcing. Ann. of Math. (2), 164(3):9931032, 2006.

[10] N. Ikeda and S. Watanabe. Stochastic differential equations and diffusion processes, volume 24 of North-Holland Mathematical Library. NorthHolland Publishing Co., Amsterdam; Kodansha, Ltd., Tokyo, second edition, 1989.

[11] O. Kallenberg. Foundations of modern probability. Probability and its Applications (New York). Springer-Verlag, New York, second edition, 2002 . 
[12] J. L. Menaldi and S. S. Sritharan. Impulse control of stochastic NavierStokes equations. Nonlinear Anal., 52(2):357-381, 2003.

[13] R. Temam. Navier-Stokes equations: Theory and numerical analysis. North-Holland Publishing Co., Amsterdam-New York-Oxford, 1977. Studies in Mathematics and its Applications, Vol. 2.

[14] J. Zhai and T. Zhang. Large deviations for 2-D stochastic Navier-Stokes equations driven by multiplicative Lévy noises. Bernoulli, 21(4):23512392, 2015. 